\title{
Stromal cell cadherin-11 regulates adipose tissue inflammation and diabetes
}

\author{
Sook Kyung Chang, ${ }^{1}$ Ayano C. Kohlgruber, ${ }^{1}$ Fumitaka Mizoguchi,, Xavier Michelet,, Benjamin J. Wolf, ${ }^{1}$ Kevin Wei, ${ }^{1}$ Pui Y. Lee, ${ }^{2}$ \\ Lydia Lynch, ${ }^{1,3}$ Danielle Duquette, ${ }^{3}$ Victòria Ceperuelo-Mallafré, ${ }^{3,4,5}$ Alexander S. Banks, ${ }^{3}$ and Michael B. Brenner ${ }^{1}$ \\ 'Division of Rheumatology, Immunology, and Allergy, Brigham and Women's Hospital and Harvard Medical School, Boston, Massachusetts, USA. Division of Immunology, Boston Children's Hospital, \\ Boston, Massachusetts, USA. ${ }^{3}$ Division of Endocrinology, Diabetes and Hypertension, Brigham and Women's Hospital and Harvard Medical School, Boston, Massachusetts, USA. ${ }^{4}$ Hospital Universitari de \\ Tarragona Joan XXIII, Institut d'Investigació Sanitària Pere Virgili, Universitat Rovira i Virgili, Tarragona, Spain. ${ }^{5}$ Centro de Investigación Biomédica en Red de Diabetes y Enfermedades Metabólicas Asociadas, \\ Instituto de Salud Carlos III, Madrid, Spain.
}

\begin{abstract}
M2 macrophages, innate lymphoid type 2 cells (ILC2s), eosinophils, Tregs, and invariant NK T cells (iNKT cells) all help to control adipose tissue inflammation, while M1 macrophages, TNF, and other inflammatory cytokines drive inflammation and insulin resistance in obesity. Stromal cells regulate leukocyte responses in lymph nodes, but the role of stromal cells in adipose tissue inflammation is unknown. PDGFR $\alpha^{+}$stromal cells are major producers of IL-33 in adipose tissue. Here, we show that mesenchymal cadherin-11 modulates stromal fibroblast function. Cadherin-11-deficient mice displayed increased stromal production of IL-33, with concomitant enhancements in ILC2s and M2 macrophages that helped control adipose tissue inflammation. Higher expression levels of IL-33 in cadherin-11-deficient mice mediated ILC2 activation, resulting in higher IL-13 expression levels and M2 macrophage expansion in adipose tissue. Consistent with reduced adipose tissue inflammation, cadherin-11-deficient mice were protected from obesity-induced glucose intolerance and adipose tissue fibrosis. Importantly, anti-cadherin-11 mAb blockade similarly improved inflammation and glycemic control in obese WT mice. These results suggest that stromal fibroblasts expressing cadherin-11 regulate adipose tissue inflammation and thus highlight cadherin-11 as a potential therapeutic target for the management of obesity.
\end{abstract}

\section{Introduction}

Obesity and metabolic syndrome are increasing rapidly, as overnutrition has become a major problem in modern society. Adipose tissue inflammation is a major driver of insulin resistance and metabolic syndrome in obesity $(1,2)$. Macrophages are the key cell type mediating adipose tissue inflammation $(3,4)$. Macrophages have functional plasticity, and their proinflammatory or antiinflammatory phenotypes (M1 and M2, respectively) are complex and can be altered depending on the tissue microenvironment (5). Inflammatory M1 macrophages accumulate in obese adipose tissue and produce proinflammatory cytokines such as TNF and IL-1 $\beta$. Macrophage-specific deletion of key signaling components such as JNK or I $\mathrm{I} B$ kinase $\beta$ involved in inducing proinflammatory cytokines improves obesity-induced adipose tissue inflammation and insulin resistance $(2,6)$. In contrast, alternatively activated M2 macrophages attenuate adipose tissue inflammation and obesity-induced insulin resistance (7). M2 macrophages display increased expression of CD206, CD301, arginase 1 (ARG1), and antiinflammatory factors such as IL-10. The Th2 cytokines IL-4

Conflict of interest: M.B. Brenner is a consultant for Roche, and M.B. Brenner, S.K. Chang, and L. Lynch have filed a patent describing targeting cadherin-11 as a new approach for treating type 2 diabetes and metabolic syndrome (US provisional application No. 62/091,709 and international patent PCT/US2015/065751).

Submitted: February 3, 2016; Accepted: June 8, 2017

Reference information: / Clin Invest. 2017;127(9):3300-3312.

https://doi.org/10.1172/JCI86881 and IL-13 mediate M2 macrophage differentiation $(8,9)$. A recent study using IL-13 reporter mice showed that IL-13 is predominantly produced in adipose tissue by innate lymphoid type 2 cells (ILC2s) compared with IL-13 production by CD $4^{+} \mathrm{T}$ cells or invariant NK T cells (iNKT cells) in adipose tissue (9). ILC2s in adipose tissue also mediate IL-4 production by activating eosinophils through IL-5 (9). In addition, ILC2s improve insulin sensitivity by increasing beige fat differentiation $(10,11)$. ILC2s respond to the cytokines IL-33, TSLP, and IL-25 (12). In particular, IL-33 induces the activation and expansion of ILC2s to express IL-13 and IL-5, which subsequently induces M2 macrophage and eosinophil accumulation and regulates Treg homeostasis in adipose tissue $(9,13,14)$. IL-33 administration improves glucose tolerance and accumulation of M2 macrophages in adipose tissue of $o b / o b$ mice (15). Thus, IL-33 plays a protective role in adipose tissue inflammation in obesity.

Fibroblasts are best known for their roles in matrix remodeling, which, in pathological states, can result in tissue destruction or fibrosis (16-18). However, in tissues like lymph nodes, fibroblastic stromal cells control $\mathrm{T}$ cell responses in the paracortex. Under basal conditions, they produce homeostatic CCL19, CCL21, and IL-7, which support T cell accumulation and survival $(19,20)$. Then, following activation, they suppress $\mathrm{T}$ cell responses via the production of nitric oxide (21-23). In some autoimmune inflammatory disorders such as rheumatoid arthritis (RA), fibroblasts are major producers of IL-6 as well as a range of other cytokines and chemokines (24-26). Thus, a key role for 
A

C
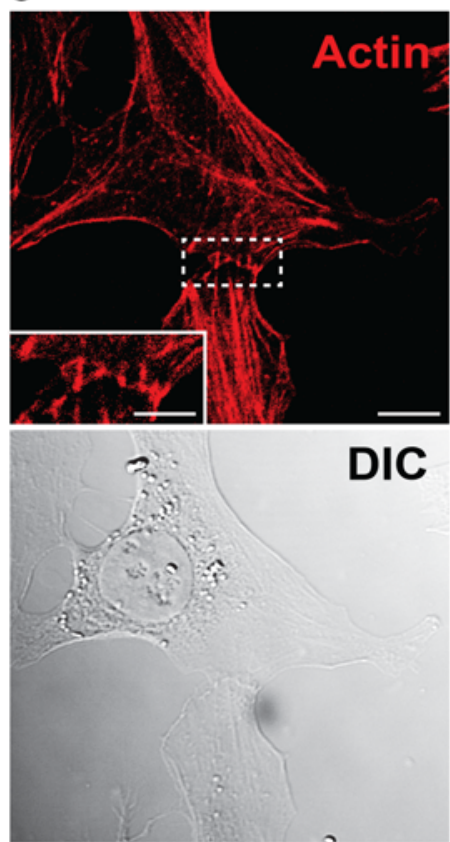

B
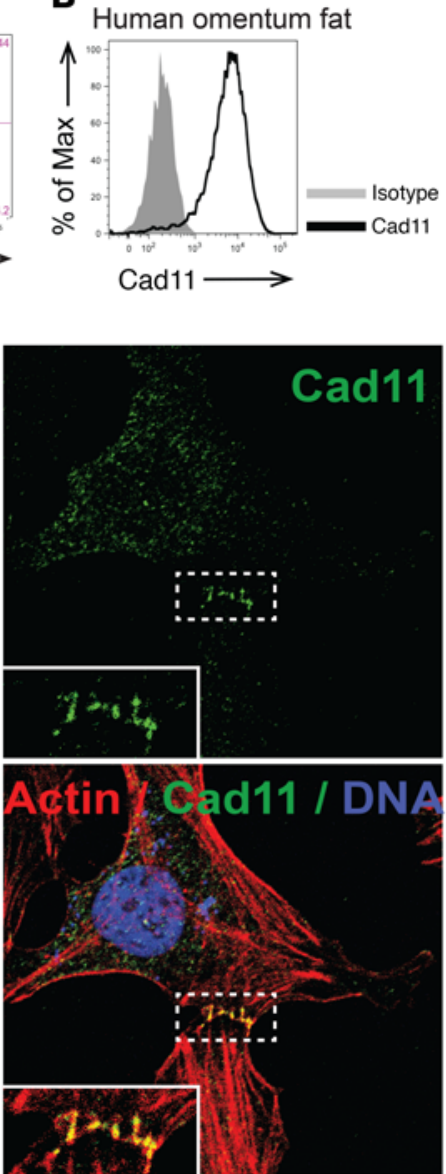
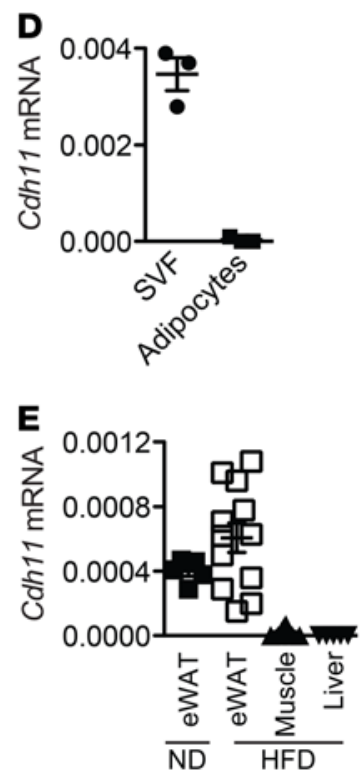

Figure 1. Cadherin-11 is expressed by fibroblasts in adipose tissue. (A) Representative flow cytometric plots of cell-surface cadherin-11 (Cad11) expression on CD45-Ter119-CD31-PDGFR ${ }^{+}$fibroblasts among SVF cells in eWAT from WT and cad-11-1- mice. (B) Cell-surface cadherin-11 expression on CD45-CD235 $\alpha$ CD31- cells in stromal vascular cells isolated from obese human omentum fat (data from 1 of 3 experiments with similar results are shown). Max, maximum. (C) Confocal microscopic images of cadherin-11 expression (green) at adherens junctions on day-2 ex vivo SVF cell cultures. Cells were costained with phalloidin (actin, red) and DRAQ5 (nucleus, blue). Scale bars: $10 \mu \mathrm{m}$ and 5 $\mu \mathrm{m}$ (insets). DIC, differential interference contrast. (D) Cdh11 mRNA relative to GAPDH in SVF cells and adipocytes from adipose tissue of WT mice $(n=3)$ fed a HFD for 5 weeks. (E) Cadherin-11 expression in eWAT, muscle, and liver from WT mice fed a ND or HFD for 5 weeks ( $n=5 \mathrm{ND}$-eWAT, $n=12 \mathrm{HFD}$ eWAT, $n=10 \mathrm{HFD}$-muscle, and $n=5$ HFD-liver; data are combined from 3 independent experiments). fibroblasts as both drivers and regulators of inflammation in specific tissue sites is emerging. However, the role of fibroblasts in adipose tissue inflammation, glycemic control, and metabolic syndrome is poorly defined. The lack of specific fibroblast markers and selectively expressed regulators of their function have been major challenges in studying and therapeutically targeting fibroblasts. Cadherin- 11 is a classical mesenchymal cadherin that mediates the homotypic cell-to-cell adhesion that is important in tissue morphogenesis and architecture $(27,28)$. Cadherin-11 is expressed by fibroblasts and myofibroblasts in a variety of tissues such as skin, lung, and joint synovium, and its expression can be induced by inflammatory stimulation $(18,29-31)$. Interestingly, targeting cadherin-11 by genetic deletion or mAb blockade has been shown to abrogate pathological fibroblast behavior in mouse models of destructive inflammatory arthritis and skin and lung fibrosis $(18,29,30)$.

Using cadherin-11 as a marker and functional modulator of fibroblasts, we show that fibroblastic stromal cells in adipose tissue regulate macrophage phenotype and glucose intolerance in diet-induced obesity. We found that cadherin-11-deficient mice had increased IL-33-producing PDGFR $\alpha^{+}$fibroblasts, increased ILC2s and IL-13, and an associated expansion of M2 macrophages. Reduced adipose tissue inflammation in cadherin-11deficient mice was associated with improved glycemic control and metabolic syndrome. Similar findings were noted after anticadherin-11 mAb therapy in WT mice.

\section{Results}

Cadherin-11 is expressed by fibroblasts in adipose tissue. Adipocytes can be separated from the stromal vascular fraction (SVF) cells that contain the leukocytes and stromal cells after enzymatic digestion of adipose tissue. Surface expression of cadherin-11 was detected by flow cytometry on nonhematopoietic stromal cells in the adipose tissue of WT mice in comparison with cad-11/-- $(\mathrm{KO})$ mice (Supplemental Figure 1; supplemental material available online with this article; https://doi.org/10.1172/JCI86881DS1). Given their spindle shape in ex vivo tissue culture and cellsurface markers, we refer here to $\mathrm{CD} 45^{-}$Ter $119^{-} \mathrm{CD} 31^{-} \mathrm{Sca}-1^{+}$ PDGFR $\alpha^{+}$cells (nonhematopoietic, non-red blood cells, nonendothelial cells) as adipose tissue fibroblasts. This fibroblast population constitutes over $90 \%$ of nonhematopoietic $\mathrm{CD}^{4} 5^{-}$ stromal vascular cells, and it includes fibroblasts, mesenchymal stem cells, adipocyte precursors, and preadipocytes. We detected cell-surface expression of cadherin-11 on approximately $10 \%$ to $50 \%$ of total adipose tissue fibroblasts (Figure $1 \mathrm{~A}$ and Supplemental Figure 1). Similarly, we found that cadherin-11 was also highly expressed by a fibroblast population of stromal vascular cells $\left(\mathrm{CD} 45^{-} \mathrm{CD} 235 \mathrm{a}^{-} \mathrm{CD} 31^{-}\right)$in the omentum of obese humans (Figure 1B). Confocal microscopic analysis further confirmed a typical staining pattern of zipper-like adhesion structures of cadherin-11 at adherens junctions between cells in ex vivo stromal vascular cell cultures (Figure 1C) (32). We further analyzed cadherin-11 expression in epididymal white adipose tissue (eWAT) by quanti- 
B

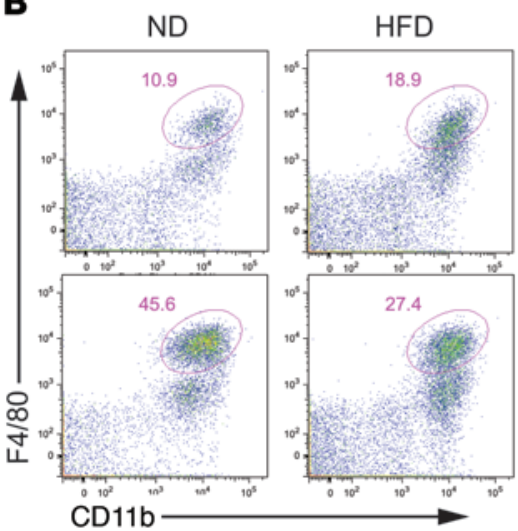

A
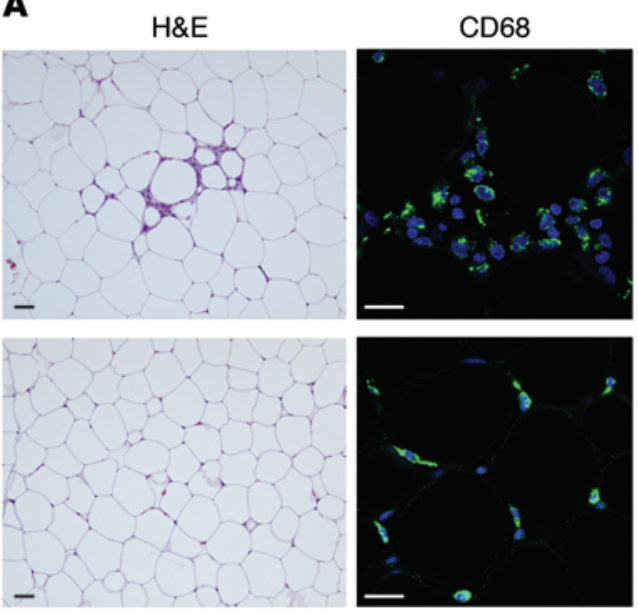

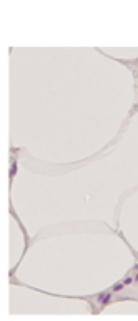

CtI

$\mathrm{F} 4 / 80$

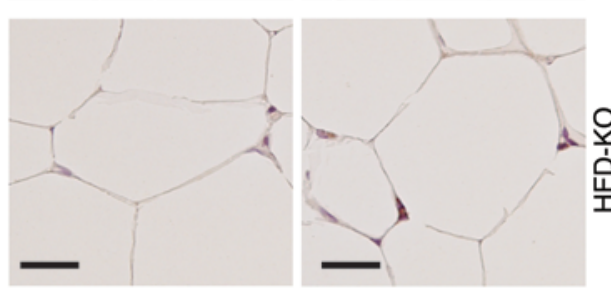

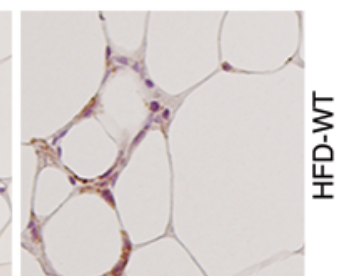
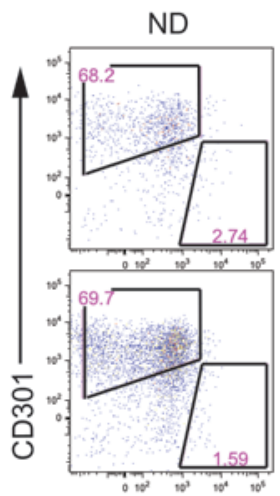

CD11c

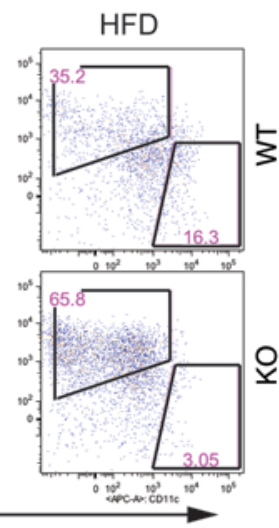

Figure 2. Reduced inflammation and increased M2 macrophages in adipose tissue of obese cad-11-/- mice. WT and cad-11 $11^{-1-}$ mice were fed a HFD for 12 weeks, unless otherwise indicated. (A) Representative fluorescence microscopic images with H\&E and anti-CD68 macrophage whole-mount staining (scale bars: $20 \mu \mathrm{m}$ ), and $\mathrm{IHC}$ with staining for isotype control (Ctl) and F4/80 (scale bars: $10 \mu \mathrm{m}$ ) in eWAT. (B) Representative flow cytometric analysis for adipose tissue macrophages in the CD45 $5^{+}$gate of SVF cells (left panel) and the $\mathrm{F} 4 / 80^{\text {hicD }} \mathrm{C1} \mathrm{b}^{+}$gate of macrophages (right panel). (C) Percentage and number of $F 4 / 80^{\text {hi }} C D 11 b^{+}$macrophages (total), CD301+CD11C ${ }^{-}$macrophages among total macrophages (M2), and CD301-CD11c ${ }^{+}$ macrophages among total macrophages (M1) in eWAT ( $n=4$ ND-WT, $n=3 \mathrm{ND}-\mathrm{KO}, n=5 \mathrm{HFD}-\mathrm{WT}$, and $n=5$ HFD-KO). Data are representative of more than 3 independent experiments. ATMs, adipose tissue macrophages. (D) QPCR analysis of the indicated genes in adipose tissue from mice fed a ND ( $n=10 \mathrm{ND}-\mathrm{WT}$ and $n=10 \mathrm{ND}-\mathrm{KO}$; pooled from 2 independent experiments) or a HFD ( $n=6$ HFD-WT and $n=5$ HFD-KO; 1 of 3 independent experiments) for 10 weeks. Data are expressed as the mean \pm SEM. ${ }^{*} P<0.05,{ }^{* *} P<0.01$, and ${ }^{* * *} P<0.001$, by 2 -way ANOVA (C) and unpaired Student's $t$ test (D).

C
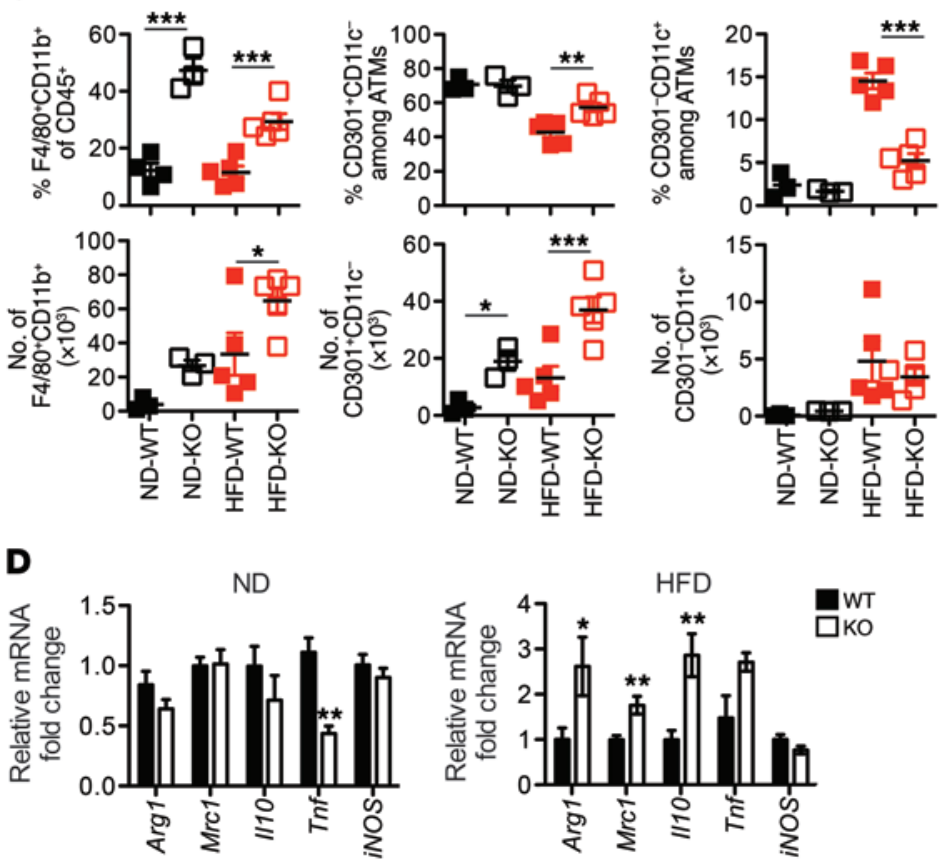

tative reverse transcription PCR (qPCR) and found that its expression was localized to the SVF, but not to mature adipocytes (Figure 1D). Compared with lean mice fed a normal chow diet (ND), cadherin-11 (Cdh11) mRNA expression tended to be higher in high-fat diet-fed (HFD-fed) WT mice, but did not reach significance (Figure 1E). We found that cell-surface cadherin-11 expression on adipose tissue fibroblasts was similar between lean and obese mice (data not shown). Compared with eWAT, cadherin-11 expression 


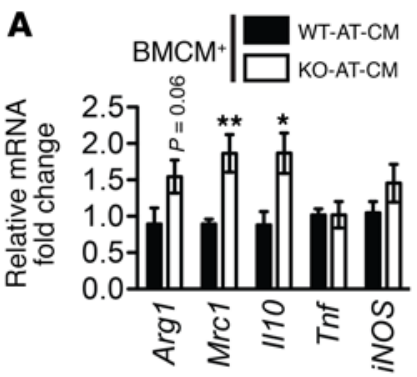

C

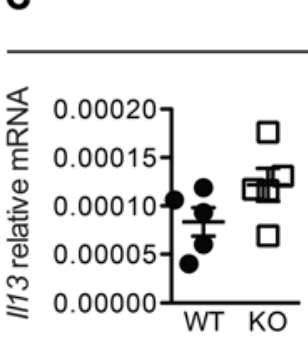

ND

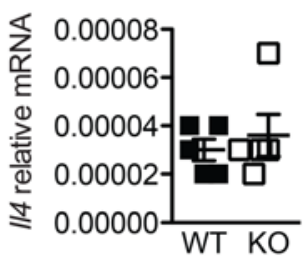

F

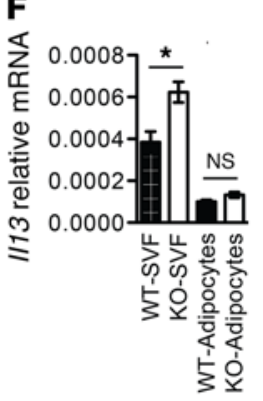

a
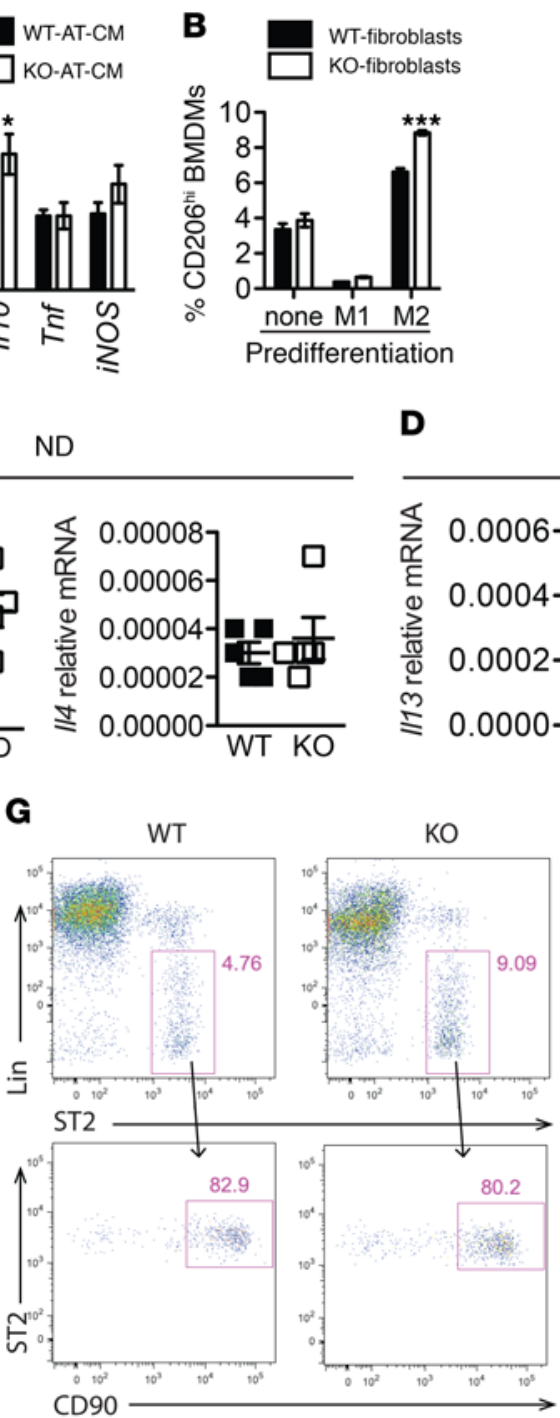

D

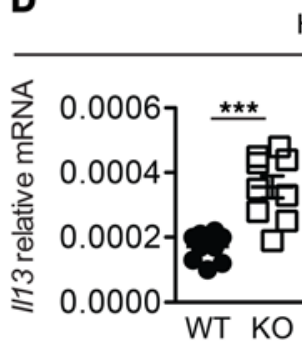

H

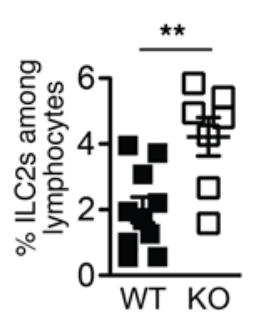

E

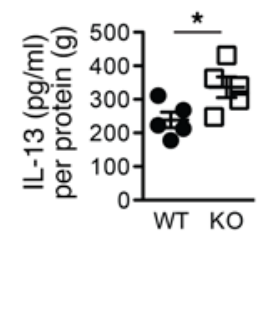

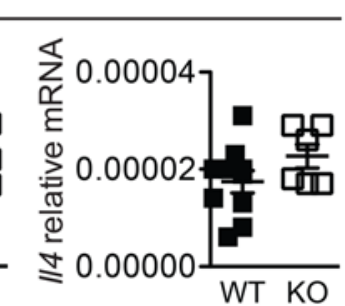

I

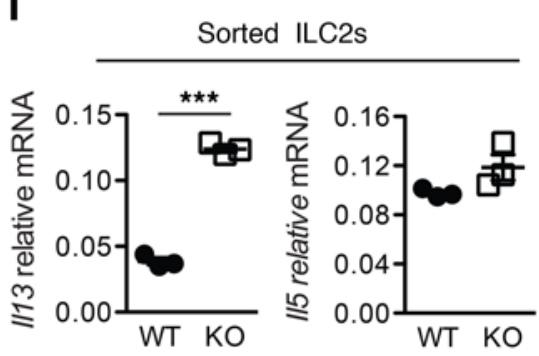

Figure 3. The levels of IL-13 produced by ILC2s are significantly higher in adipose tissue of obese cad-11/- mice than in that of WT mice. (A) qPCR analysis of pro- and antiinflammatory genes in BMDMs cultured in AT-CM for 3 days. For each experiment, AT-CM was obtained from the adipose tissue of WT and cad-11 $11^{--}$mice fed a HFD for 5 weeks ( $n=3$ HFD-WT and $n=3$ HFD-KO). Data are representative of 3 independent experiments. (B) BMDMs were untreated or predifferentiated into either M1 or M2 macrophages. After direct coculturing with fibroblasts derived from adipose tissue of WT or cad-11-/mice, the percentage of surface CD206 $6^{+}$BMDMs was analyzed by flow cytometry (data from 1 of 3 experiments with similar results are shown, and in each experiment, different fibroblast lines derived from WT or cad-11 ${ }^{-1-}$ mice were used). (C and D) Comparison of mRNA levels of $I / 13$ and $I / 4$ in adipose tissue from WT and cad-11-/- mice fed a ND (C, $n=5$ ND-WT and $n=5$ ND-KO) or a HFD (D, left graph: $n=10$ HFD-WT and $n=9$ HFD-KO; D, right graph: $n=10$ HFDWT and $n=6$ HFD-KO; data were pooled from 2 independent experiments). (E) IL-13 protein in adipose tissue from HFD-fed mice was detected by ELISA ( $n=5 \mathrm{HFD}-\mathrm{WT}$ and $n=5 \mathrm{HFD}-\mathrm{KO}$ ). Data are representative of 2 independent experiments. (F) $/ 113 \mathrm{mRNA}$ expression in SVF cells and adipocytes fractioned after digestion of adipose tissue from HFD-fed mice ( $n=4$ WT-SVF, $n=6$ KO-SVF, $n=5$ WT-adipocytes, and $n=7$ KO-adipocytes; data were pooled from 2 independent experiments). (C) Representative flow cytometric analysis of ILC2s in adipose tissue. (H) Percentage of ILC2s among CD45+ lymphocytes in adipose tissue of WT and cad-11 ${ }^{-/-}$mice fed a HFD for 5 weeks ( $n=10 \mathrm{WT}$ and $n=7 \mathrm{KO}$; data were pooled from 2 independent experiments). (I) mRNA levels of $/ 113$ and $/ / 5$ in flow-isolated ILC2s from adipose tissue of WT and cad-11-/- mice fed a HFD for 12 weeks $(n=3$ HFD-WT and $n=3$ HFD-KO). Data are expressed as the mean $\pm \mathrm{SEM} .{ }^{*} P<0.05,{ }^{* *} P<0.01$, and ${ }^{* * *} P<0.001$, by unpaired Student's $t$ test $(\mathbf{A}, \mathbf{B}, \mathbf{D}-\mathbf{F}, \mathbf{H}$, and $\mathbf{I})$.

was very low, at or below the level of detection in muscle and liver of HFD-fed WT mice (Figure 1E). These data demonstrate that cadherin-11 is expressed on fibroblast populations but not on mature adipocytes, hematopoietic cells, or endothelial cells in the WAT of mice and humans.

cad-11 $11^{--}$mice have reduced adipose tissue inflammation and increased M2 macrophages in diet-induced obesity. The appearance of crown-like structures composed of clusters of M1 macrophages and other inflammatory cells is a hallmark of adipose tissue inflam- mation in obesity. Following HFD feeding, crown-like structures accumulating macrophages were visualized in adipose tissue by immunofluorescence microscopy with staining for $\mathrm{CD}^{+} 8^{+}$and by IHC with staining for $\mathrm{F} 4 / 80$. We found that obese cad-11/-- mice had substantially fewer crown-like structures than did obese WT mice (Figure 2A). In contrast to the smaller number of crownlike structures, we detected a higher percentage and number of total macrophages in obese cad11 $11^{-/}$mice (Figure 2, B and C). However, M2 macrophages $\left(\mathrm{CD} 301^{+} \mathrm{CD} 11 \mathrm{c}^{-} \mathrm{F} 4 / 80^{+} \mathrm{CD} 11 \mathrm{~b}^{+}\right.$cells) 

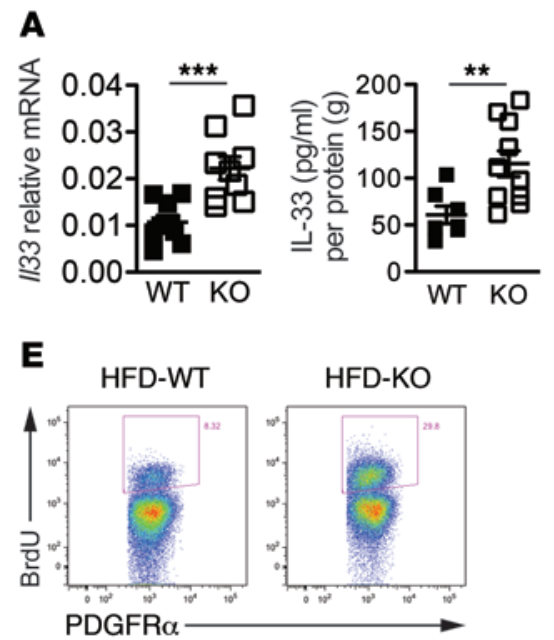

B

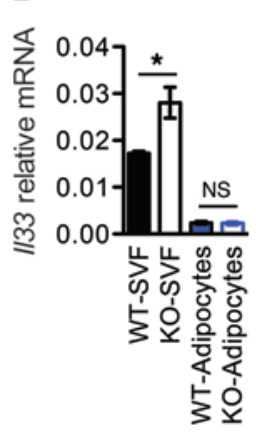

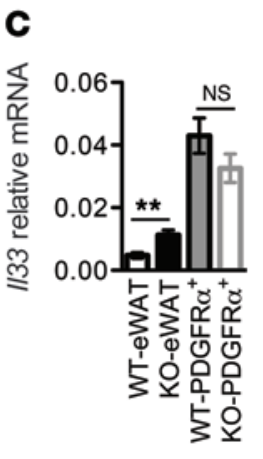

D
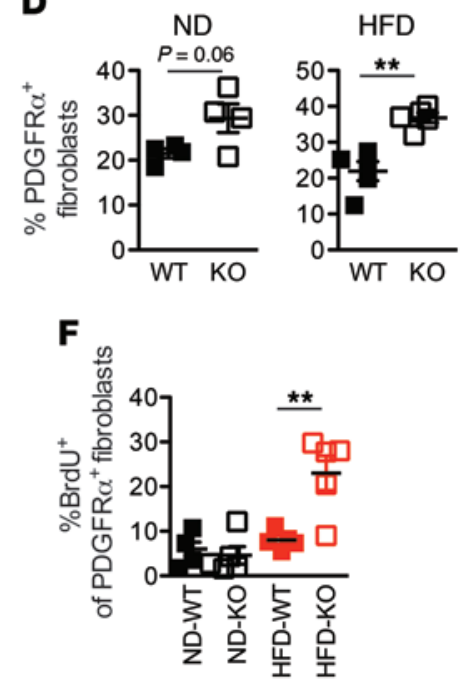

Figure 4. PDGFR $\alpha^{+}$fibroblast expansion accounts for higher expression of IL-33 in cad-11 ${ }^{-/-}$adipose tissue. (A) IL-33 expression in adipose tissue of WT and cad-11 $1{ }^{-1}$ mice fed a HFD for 5 weeks (left graph: $n=9$ HFD-WT and $n=9$ HFD-KO, pooled from 2 independent experiments; right graph: $n=7$ HFD-WT and $n=10 \mathrm{HFD}-\mathrm{KO}$, pooled from 2 independent experiments). (B) I/33 mRNA expression in SVF cells and adipocytes isolated from adipose tissue of mice fed a HFD for 12 weeks ( $n=4$ WT-SVF, $n=6 \mathrm{KO}-\mathrm{SVF}, n=5 \mathrm{WT}$-adipocytes, and $n=7 \mathrm{KO}$-adipocytes, pooled from 2 independent experiments). (C) I/33 mRNA expression in adipose tissue and flow-isolated PDGFR $\alpha^{+}$fibroblasts $\left(n=5\right.$ WT-eWAT, $n=5 \mathrm{KO}$-eWAT, $n=5 \mathrm{WT}$-PDGFR $\alpha^{+}$, and $n=5 \mathrm{KO}$-PDGFR $\left.\alpha^{+}\right)$. Data are representative of 2 independent experiments. (D) Percentage of PDCFR $\alpha^{+}$fibroblasts in SVF cells from mice fed a ND or HFD for 5 weeks ( $n=4$ ND-WT, $n=4$ ND-KO, $n=5 \mathrm{HFD}-\mathrm{WT}$, and $n=5 \mathrm{HFD}-\mathrm{KO}$ ). Results are representative of more than 3 independent experiments. (E) Representative flow cytometric plots of BrdU uptake in PDGFR $\alpha^{+}$fibroblasts from WT and cad-11 $11^{-/}$mice fed a HFD for 1 week. (F) Percentage of BrdU ${ }^{+}$cells among PDGFR $\alpha^{+}$fibroblasts $(n=5$ ND-WT, $n=5$ ND-KO). Results are representative of 2 independent experiments. Data are expressed as the mean $\pm \mathrm{SEM} .{ }^{*} P<0.05,{ }^{* *} P<0.01$, and ${ }^{* * *} P<0.001$, by unpaired Student's $t$ test (A-D and $\mathbf{F}$ ).

were the major macrophage population in adipose tissue from both lean and obese cad-11/-- mice. The percentage of M1 macrophages $\left(\mathrm{CD} 301^{-} \mathrm{CD} 11 \mathrm{c}^{+} \mathrm{F} 4 / 80^{+} \mathrm{CD} 11 \mathrm{~b}^{+}\right.$cells) was significantly decreased, but these percentages were similar in adipose tissue of obese cad- $11^{-/-}$mice compared with that of obese WT mice (Figure 2, B and $\mathrm{C}$ ). The mRNA expression of M2 macrophage markers such as Arg1, Mrc1, and Il1O was also substantially higher in adipose tissue of obese cad- $11^{-/-}$mice (Figure 2D). These results indicate that there were increased numbers of M2, but not M1, macrophages in adipose tissue of cad-11/- mice. Myeloid cell lineage development in bone marrow and monocyte populations (Ly6 $\mathrm{C}^{\text {hi }} \mathrm{CXCR} 3^{+}$and $\mathrm{Ly} 6 \mathrm{C}^{\mathrm{lo}}$

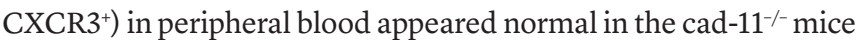
(Supplemental Figure 2, A and B). These results suggested that the increased M2 macrophage numbers in cad- $11^{-/-}$mice were likely a result of the local adipose tissue microenvironment. Taken together, these findings suggested that cad- $11^{-/-}$fibroblasts may regulate the adipose tissue macrophage phenotype.

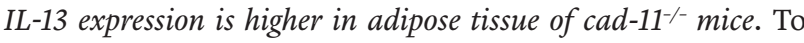
determine whether cadherin-11 deficiency is responsible for M2 macrophage differentiation, we examined bone marrow-derived macrophages (BMDMs) treated with conditioned medium from ex vivo cultures of adipose tissue from either WT or cad-11/mice fed a HFD. Under these conditions, BMDMs cultured with cad- $11^{-/-}$adipose tissue-derived conditioned medium (KO-ATCM) expressed higher levels of mRNA for M2 macrophageassociated markers (Mrc1, Arg1, and Il1O), but not M1 macrophageassociated markers (Tnf and $i N O S$ ), compared with WT adipose tissue-derived conditioned medium (WT-AT-CM) (Figure 3A). The results using WT BMDMs were consistent with our previous analyses of increased expression of the M2 markers Arg1, Mrc1, and $I l 10$, but no changes in the M1 macrophage markers Tnf or iNOS in adipose tissue of obese cad-11/- mice (Figure 2D). These data indicate that M2 macrophage differentiation is mediated by soluble factor(s) in cad-11/-- adipose tissue.

To confirm that fibroblasts in cad-11/-- adipose tissue mediate macrophage differentiation, we prepared cocultures of adipose tissue-derived fibroblasts with BMDMs in vitro. WT and cad-11/fibroblasts showed similar effects on the levels of CD206, a M2 macrophage marker expressed in BMDMs (Figure 3B), suggesting that cad-11/- fibroblasts do not directly produce soluble factor(s) for macrophage polarization into M2 macrophages. However, cad-11/- fibroblasts significantly enhanced the surface expression of CD206 on BMDMs predifferentiated into M2 mac-

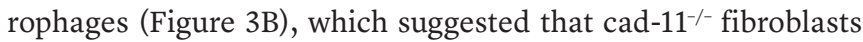
promote the M2 macrophage phenotype instead of initiating M2 macrophage polarization.

Next, we examined IL-13 and IL-4, factors that are well known in M2 macrophage differentiation. The levels of both IL-13 and IL-4 were similar in adipose tissue of lean WT and cad-11/- mice, with a slight trend toward higher Il13 levels in cad-11/-- adipose tissue (Figure 3C). However, Il13 mRNA expression was significantly higher in the adipose tissue of obese cad-11/ ${ }^{--}$mice compared with mRNA levels in similarly obese WT mice (Figure 3D). Higher IL-13 expression at the protein level was confirmed by ELISA in adipose tissue of obese

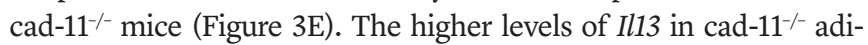
pose tissue were detected in SVF cells, but not in adipocytes (Figure $3 F)$. These results suggested that higher expression of IL-13 in obese cad- $11^{-/-}$adipose tissue induces M2 macrophage differentiation. 

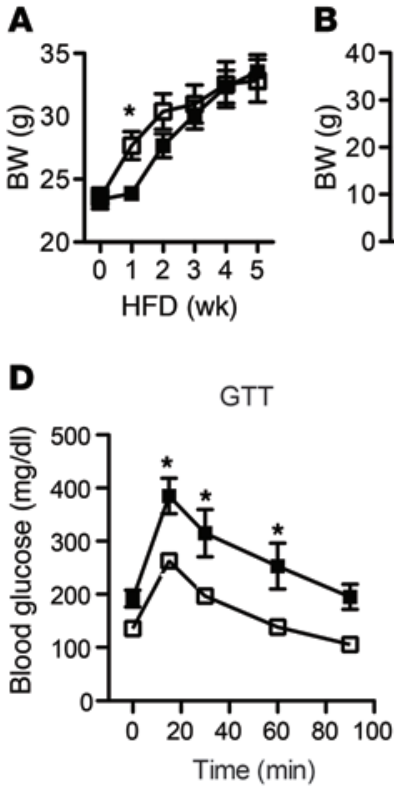

$\mathbf{F}$

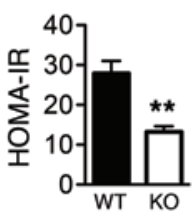

G
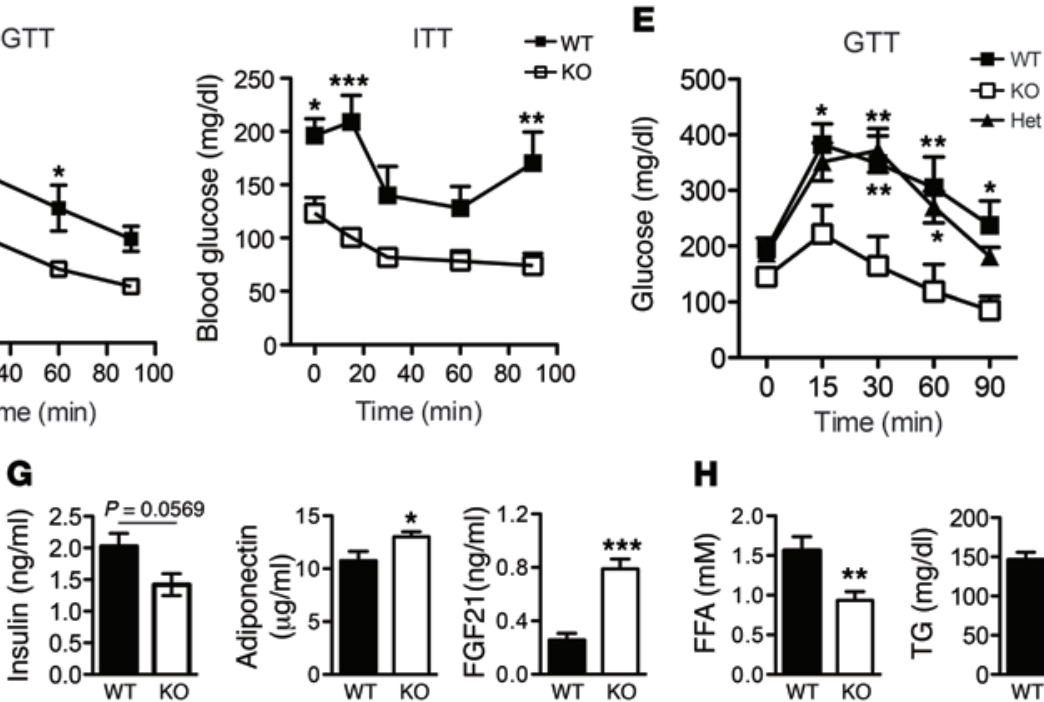

H
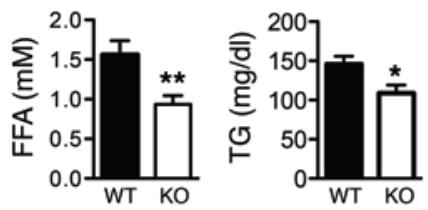

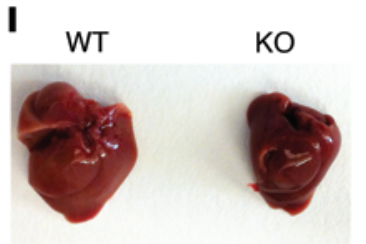

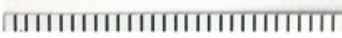
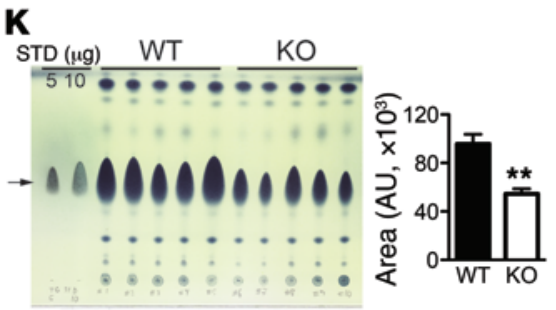

J
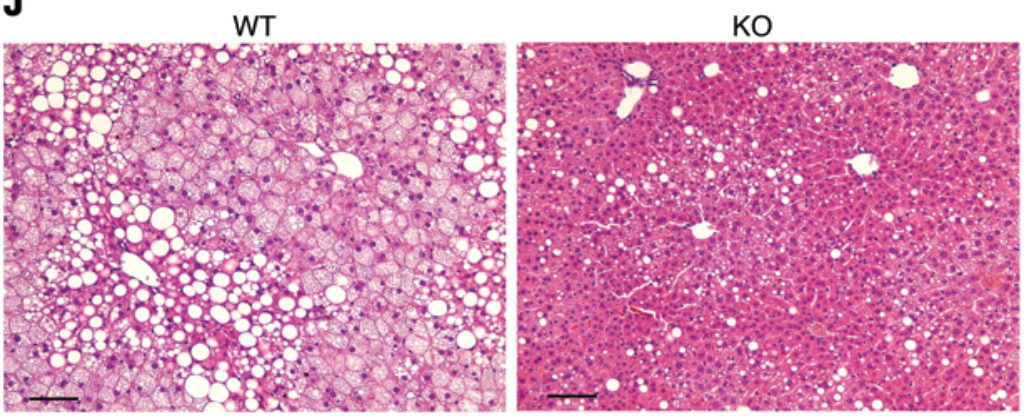

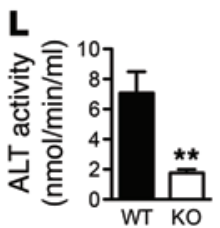

Figure 5. Cadherin-11 ${ }^{-/-}$deficiency protects obese mice from poor glycemic control. WT and cad-11-/- mice were fed a HFD for 5 weeks, unless otherwise indicated. (A) BW of mice on a HFD ( $n=5$ HFD-WT and $n=4$ HFD-KO). Data are representative of more than 3 independent experiments. EchoMRI analysis for (B) BW and (C) lean and fat mass ( $n=7 \mathrm{ND}-\mathrm{WT}, n=5 \mathrm{ND}-\mathrm{KO}, n=5 \mathrm{HFD}-\mathrm{WT}$, and $n=4$ HFD-KO). Data are representative of 2 independent experiments. (D) GTTs and ITTs for WT and cad-11-/- mice fasted overnight for the GTT ( $n=9$ HFD-WT and $n=8$ HFD-KO) and fasted 4 hours for the ITT ( $n=4$ HFD-WT and $n=5$ HFD-KO). Data are representative of more than 3 independent experiments. (E) GTTs for littermates of WT and cad-11 $11^{-/-}$mice fed a HFD for 5 weeks ( $n=7$ HFD-WT, $n=7$ HFD-Het, and $n=5$ HFD-KO). Het, heterozygous. (F) HOMA-IR index. (C) Serum levels of insulin ( $n=5$ HFD-WT and $n=5$ HFD-KO), adiponectin, and FGF21 ( $n=8-9$ per group, pooled from 2 independent experiments). (H) Serum levels of FFA ( $n=10$ per group) and TC ( $n=17-18$ per group, pooled from 3 independent experiments). Serum factor analyses were done separately with serum samples collected from 3 independent experiments. (I) Representative image of livers and graph showing liver weights for WT and cad-11 $1^{-/-}$mice $(n=10$ per group, combined from 2 independent experiments). (J) Representative H\&E staining of liver sections from WT and cad-11 $11^{-/-}$mice fed a HFD for 12 weeks. Scale bars: $100 \mu \mathrm{m}$. (K) TLC analyses of nonpolar lipids isolated from livers of obese WT and cad-11/-/- mice. Arrow indicates the TG-specific bands as determined by the TC standards (STD) on the TLC plate. Graph shows Imagel densitometric analysis of TLC-resolved TC ( $n=5$ HFD-WT and $n=5$ HFD-KO). Data are representative of 2 independent experiments. (L) Analysis of ALT activity in serum collected from mice fed a HFD for 12 weeks ( $n=7$ HFD-WT and $n=6$ HFD-KO, combined from 2 independent experiments). Data are expressed as the mean \pm SEM. ${ }^{*} P<0.05$, ${ }^{* *} P<0.01$, and ${ }^{* *} P<0.001$, by unpaired Student's $t$ test $(\mathbf{A}-\mathbf{C}, \mathbf{F}-\mathbf{I}, \mathbf{K}$, and $\mathbf{L})$ and 2 -way ANOVA, for WT and cad-11 $11^{-1-}$ mice ( $\mathbf{D}$ and $\left.\mathbf{E}\right)$. 


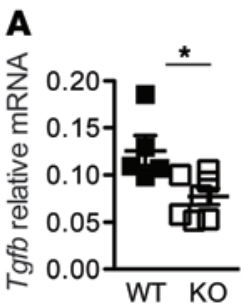

$\mathbf{B}$
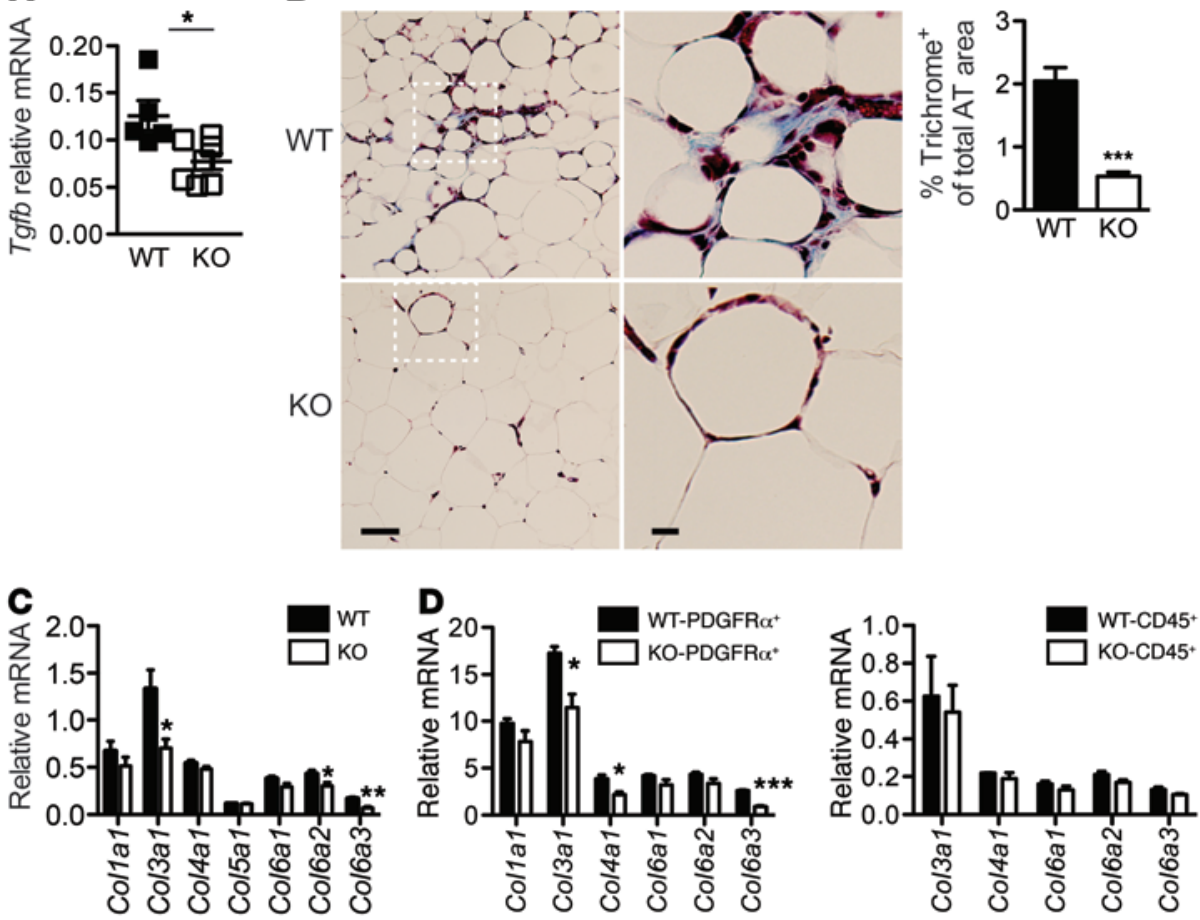

G
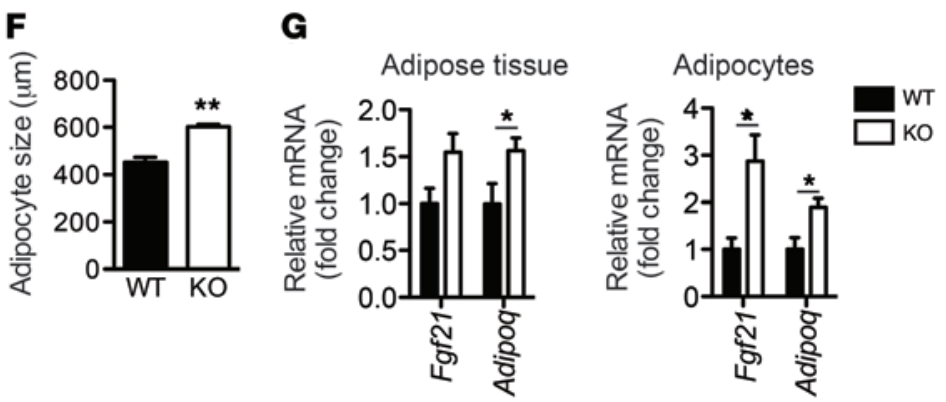

Figure 6. Less fibrotic adipose tissue of cad-11 ${ }^{-/-}$mice contains healthy adipocytes in obesity. (A) mRNA expression of Tgfb in obese adipose tissue of WT and cad-11 ${ }^{-1-}$ mice ( $n=5 \mathrm{HFD}-\mathrm{WT}$ and $n=7 \mathrm{HFD}-\mathrm{KO}$ ). Data are representative of 2 independent experiments. (B) Representative Masson's trichrome staining of adipose tissue from WT and cad-11/- mice fed a HFD for 12 weeks. Scale bars: $100 \mu \mathrm{m}$ and $20 \mu \mathrm{m}$ (insets). The percentage of blue-colored trichromepositive staining of the adipose tissue (AT) area was measured with Imagel (15 images were taken from HFD-WT [ $n=3$ ]; 11 images were taken from HFD-KO $[n=4]$ mice). Data are representative of 2 independent experiments. (C) qPCR analysis of collagens in adipose tissue ( $n=5$ HFD-WT and $n=7$ HFD-KO). Data are representative of 2 independent experiments. (D) qPCR analysis of flow-isolated PDGFR $\alpha^{+}$fibroblasts and CD45 ${ }^{+}$cells ( $n=3 \mathrm{HFD}-\mathrm{WT}$ and $n=3 \mathrm{HFD}-\mathrm{KO}$ ). Data are representative of 2 independent experiments. (E) Size distribution of adipocytes. (F) Average size of adipocytes. Adipocyte size was measured by Image) in H\&E-stained adipose tissue from WT and cad-11-1- mice fed a HFD for 5 weeks (46 images were taken from HFD-WT [ $n=4$ ]; 44 images were taken from HFD-KO $[n=6]$ mice). Data are representative of 2 independent experiments. (G) qPCR analysis of adiponectin and FGF21 in adipose tissue and isolated adipocytes from WT and cad-11-/- mice fed a HFD for 12 weeks ( $n=5 \mathrm{HFD}-\mathrm{WT}$ and $n=7 \mathrm{HFD}-\mathrm{KO}$ ). Data are representative of 2 independent experiments. Data are expressed as the mean \pm SEM. ${ }^{*} P<0.05,{ }^{* *} P<0.01$, and ${ }^{* *} P<0.001$, by unpaired Student's $t$ test $(\mathbf{A}-\mathbf{D}, \mathbf{F}$, and $\mathbf{C})$.

ILC2s are activated in adipose tissue of obese cad-11/- mice. Since IL-13 is predominantly produced by ILC2s in adipose tissue (9), we compared ILC2s in adipose tissue of obese WT and cad-11mice (Figure 3G and Supplemental Figure 3A). Indeed, we found that ILC2 numbers were significantly higher in adipose tissue of cad-11 $11^{--}$mice than in that of WT mice fed a HFD (Figure $3 \mathrm{H}$ ). Consistent with the lack of expression of cadherin-11 on CD $45^{+}$ cells shown in Supplemental Figure 1, ILC2s lacked expression of cadherin-11 (data not shown). Furthermore, Il13 expression was markedly higher in flow-sorted ILC2s from adipose tissue of obese cad-11/- compared with ILC2s from obese WT mice (Figure 3I). This suggests that the expansion and activation of ILC2s are responsible for the increased IL-13 production observed in obese cad-11-- adipose tissue. Il5 expression by flow-sorted ILC2s was similar in WT and cad-11/-- mice (Figure 3I), which is consistent with there being similar numbers of eosinophils and similar levels of Il4 expression in obese WT and cad-11-/- adipose tissue (Figure 3D and Supplemental Figure 3B). Interestingly, lean cad-11 ${ }^{--}$mice had a higher percentage of eosinophils in adipose tissue than did WT mice (Supplemental Figure 3B), which may influence the increased M2 macrophage phenotype in lean cad-11 $1{ }^{--}$mice. Together, the data suggested that ILC2-derived IL-13 mediates M2 macrophage differentiation in adipose tissue of cad-11/- mice. 
A

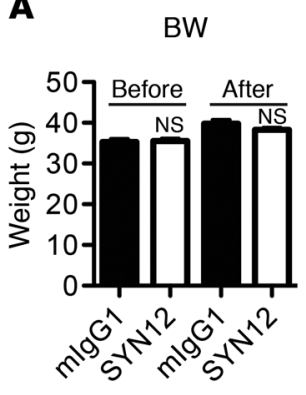

B

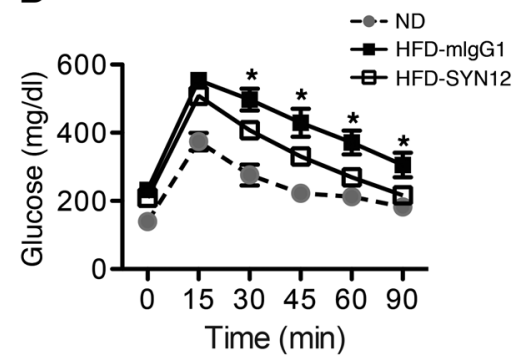

E

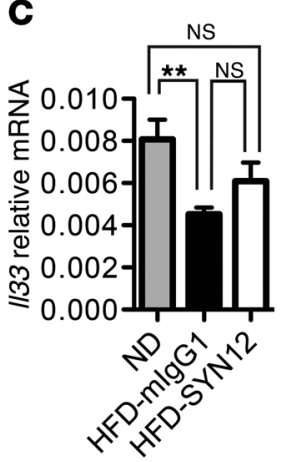

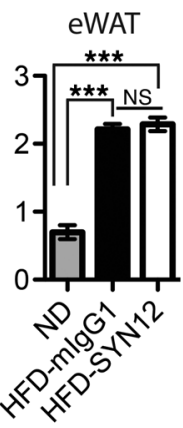

D

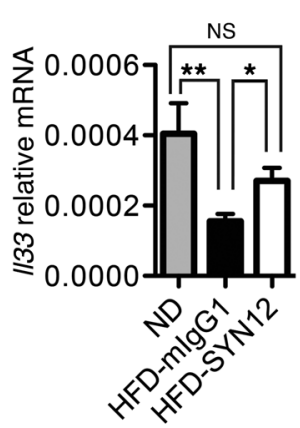

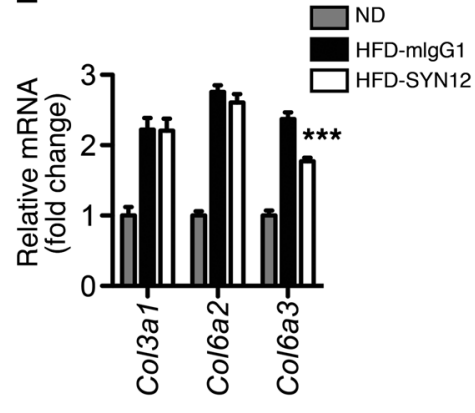

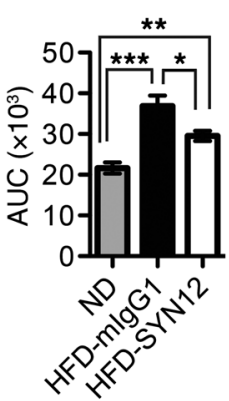

$\mathbf{F}$

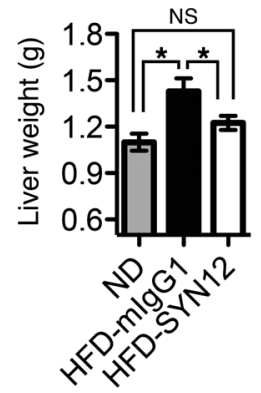

Figure 7. Blockade by anticadherin-11 mAb in obese WT mice improves glycemic control in obesity. B6 WT mice were injected i.p. with an anticadherin-11-specific Ab (SYN12) or mlgG1 isotype control Ab (10 $\mathrm{mg} / \mathrm{kg} \mathrm{BW}$ ) every 3 days for the last 3 weeks of a 9-week HFD ( $n=5 \mathrm{ND}, n=7$ mlgG1-treated HFD-fed mice, and $n=8$ SYN12-treated HFD-fed mice). Data are representative of 3 independent experiments. (A) BW before and after HFDfeeding and fat pad weights. (B) GTT and AUC data. (C-E) qPCR analysis of $/ 13,1 / 13$, and collagens in adipose tissue.

(F) Liver weights. Data are expressed as the mean \pm SEM ${ }^{*} P<0.05,{ }^{*} P<0.01$, and ${ }^{* * *} P<0.001$, by unpaired Student's $t$ test (A, B for the AUC graph, and $\mathbf{C}-\mathbf{F}$ ) and 2-way ANOVA for mlgG1-treated HFDfed mice versus SYN12-treated HFD-fed mice (B).
Cad-11/- fibroblasts produce IL-33 and are expanded in adipose tissue of obese mice. Next, we determined the mechanism by which cad- $11^{-/-}$fibroblasts mediate ILC2 activation. ILC2s are activated by IL-33, TSLP, and IL-25. Among these, the ST2 ligand IL-33 is known to induce ILC2 expansion in adipose tissue $(9,33)$. Our qPCR analysis showed that, compared with $\mathrm{CD} 45^{+}$cells, nonhematopoietic cells are the main cells expressing IL-33 (Supplemental Figure 4A). In lean mice, Il33 mRNA appeared slightly higher in the adipose tissue of cad-11/-- mice compared with that of WT mice, but did not reach significance (Supplemental Figure 4B). However, Il33 mRNA and protein expression levels were markedly higher in the adipose tissue of HFD-fed cad-11/- mice than in that of WT mice (Figure 4A). Further, the levels of Tslp, but not Il25, were also increased in adipose tissue of obese cad-11/-- mice (Supplemental Figure 4C). The increased expression of Il33 was detected in SVF cells, not adipocytes (Figure 4B). qPCR for Il33 in flow-isolated PDGFR $\alpha^{+}$fibroblasts revealed that fibroblasts from

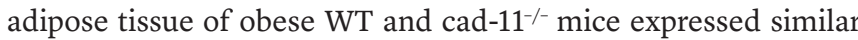
levels of Il33 and other ligands (Figure 4C and Supplemental Figure 4D). To our surprise, however, there was a significant increase in the number of PDGFR $\alpha^{+}$fibroblasts in obese cad-11/-- adipose tissue compared with that detected in obese WT mice (Figure $4 \mathrm{D}$ and Supplemental Figure 4E). PDGFR $\alpha^{+}$fibroblasts were also present at slightly higher levels in adipose tissue of lean cad-11 ${ }^{-1-}$ mice (Figure 4D), which was consistent with the slightly higher level of IL-33 expression in adipose tissue of lean cad-11/-/ mice compared with that of WT mice (Supplemental Figure 4B). Therefore, we found that the basis for the increased levels of IL-33 resulted not from the amount made per cell in cad- $11^{-/-}$versus WT fibroblasts, but rather from the significantly higher number of adipose tissue fibroblasts in cad-11//- mice. We determined whether cad $-11^{-/-}$PDGFR $\alpha^{+}$fibroblasts were increased as a result of a higher proliferation rate. After 5 weeks of HFD feeding, the proliferation rate of cad- $11^{-/-}$PDGFR $\alpha^{+}$fibroblasts $(1.992 \% \pm 0.4062 \%)$ was significantly lower than that of WT PDGFR $\alpha^{+}$fibroblasts $(3.528 \% \pm$ $0.42 \%$ ) (Supplemental Figure 4F). However, during the first week of the HFD, compared with WT mice $(8.014 \% \pm 0.9219 \%)$, a significantly higher percentage of PDGFR $\alpha^{+}$fibroblasts in adipose tissue of cad- $11^{-/-}$mice $(23.03 \% \pm 3.840 \%)$ consisted of proliferating BrdU ${ }^{+}$cells (Figure 4, E and F). These results suggested that the increased number of PDGFR $\alpha^{+}$fibroblasts in adipose tissue of cad-11/- mice results from a higher proliferation rate at the early stage of HFD feeding.

Deficiency of cadherin-11 improves insulin resistance and hepatic steatosis in obesity. Reduced crown-like structures, higher levels of Th2 cytokines, and increased numbers of M2 macrophages all indicated a substantial reduction in inflammation in the adipose tissue of obese cad- $11^{-/-}$mice. Thus, we determined whether cadherin-11-expressing fibroblasts modulate metabolic phenotypes in diet-induced obese mice. WT and cad-11/- mice showed no significant differences in daily food intake (Supplemental Figure

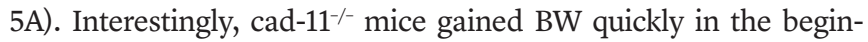
ning of the HFD, but their BW became similar to that of WT mice with continued HFD feeding (Figure 5, A and B). A trend toward increased fat pad weight was seen in cad-11//- mice (Figure $5 \mathrm{C}$ ). Compared with WT mice, HFD-fed cad-11 $1^{-/}$mice showed similar adiposity, but, surprisingly, glucose tolerance tests (GTTs) and insulin tolerance tests (ITTs) showed that cad- $11^{-/-}$mice were nearly completely protected from obesity-induced glucose intolerance (Figure 5D). Note that glucose tolerance was confirmed in cad- $11^{-/-}$littermates compared with WT mice, suggesting that the improved glucose tolerance in obese cad-11/-- mice resulted from the absence of cadherin-11, and not from potential genetic background differences (Figure 5E and Supplemental Figure 5B). In addition, 


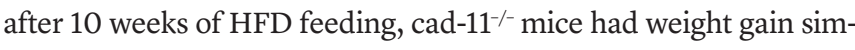
ilar to that of WT mice but still maintained nearly normal glucose tolerance (Supplemental Figure 5, C and D). The homeostatic model assessment insulin resistance (HOMA-IR) index and serum insulin levels also confirmed insulin sensitivity in the obese cad-11/-- mice (Figure 5, F and G). Serum levels of adiponectin and FGF21 were also markedly higher in HFD-fed cad-11/-- mice (Figure 5G).

Consistent with these healthy adipocyte functions and insulin sensitivity, we found lower free fatty acids (FFA) and triglycerides (TG) in the serum of HFD-fed cad-11/-- mice (Figure $5 \mathrm{H}$ ). In addition, livers from obese cad-11/- mice weighed significantly less (Figure 5I and Supplemental Figure 5E) and had markedly fewer lipid droplets than did livers from obese WT mice (Figure 5J). TLC analyses confirmed that liver TG content was significantly lower in cad-11/- mice than in WT mice fed a HFD, as quantified by the density of the TLC bands (Figure 5K). Analysis of fatty acid biosynthetic genes in liver showed that mRNA levels of ATP citrate lyase $(\mathrm{Acl})$ and fatty acid synthase (Fasn), but not acetyl-CoA carboxylase $1(A c c)$, were decreased in obese cad-11/-- mice (Supplemental Figure $5 \mathrm{~F}$ ). This suggests that de novo lipogenesis was lower and probably contributed to less fat accumulation in the livers of obese cad- $11^{-/-}$mice. Serum ALT analysis, which measures liver damage (Figure 5L), confirmed that the livers of obese cad-11/-- mice were healthier. In fact, the ALT activity was comparable to that seen in lean WT mice (data not shown). Consistent with improved glucose tolerance, compared with WT mice, we observed significantly improved insulin action in adipose tissue, liver, and

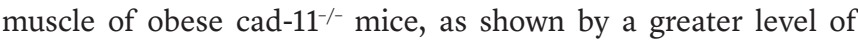
phosphorylated AKT (p-AKT) following insulin administration in vivo (Supplemental Figure 5, G and $\mathrm{H}$ ). These findings show that cad- $11^{-/-}$mice have healthy adipose tissue function and markedly less obesity-induced hepatic steatosis and glucose intolerance in diet-induced obesity compared with WT mice.

cad-11/- mice have significantly less adipose tissue fibrosis. Unresolved chronic inflammation often contributes to tissue fibrosis mediated by fibroblasts and myofibroblasts. Thus, we determined whether cad-11/- adipose tissue is protected from obesityinduced adipose tissue fibrosis. We found that TGF- $\beta$ (Tgfb) mRNA expression was markedly lower in obese adipose tissue of cad-11/- mice than in that of WT mice (Figure 6A), which was consistent with histological analysis of obese adipose tissue that showed significantly less collagen deposition (trichromepositive blue- or Sirius red-colored areas) in HFD-fed cad-11/mice compared with WT mice (Figure 6B and Supplemental Figure 6A). We also found that the expression of several key collagen genes, Col3a1, Col6a2, and Col6a3, was significantly lower in adipose tissue from obese cad-11/- mice (Figure 6C). To determine whether PDGFR $\alpha^{+}$fibroblasts were responsible for the collagen expression in adipose tissue, PDGFR $\alpha^{+}$fibroblasts were isolated either by flow cytometry or magnetic beads and found to be the predominant cells producing collagen in adipose tissue. The expression of Col3a1, Col4a1, and Col6a3 was substantially lower in cad-11/- $\mathrm{PDGFR}^{+}$fibroblasts than in WT PDGFR $\alpha^{+}$ fibroblasts, whereas $\mathrm{CD} 45^{+}$cells showed similar expression levels of these collagens (Figure 6D). In particular, the lower expression levels of Col3a1 and Col6a3 by cad-11/- PDGFR $\alpha^{+}$fibroblasts were consistent with the lower levels of their total mRNA expression in cad- $11^{-/-}$adipose tissue. These results suggest that both reduced inflammation and lower collagen production by fibroblasts result in less fibrosis in adipose tissue of obese cad-11 $11^{-/-}$mice. Interestingly, adipose tissue from obese cad- $11^{-/-}$mice showed a clearly different morphology from that of obese WT mice (Supplemental Figure 6B). Previous reports have noted that adipocytes can be larger in a flexible and less rigid adipose tissue extracellular matrix (ECM) environment $(34,35)$. Correspondingly, we found an increased percentage of larger adipocytes in HFD-fed cad-11/mice than in WT mice (Figure 6, E and F). Moreover, adipocytes

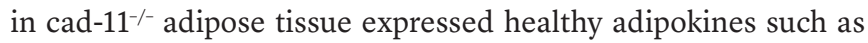
FGF21 and adiponectin (Figure 6G). Together, these data suggested that lower collagen production, particularly of COL3 and COL6, prevents adipose tissue fibrosis following HFD feeding in cad-11/- mice. This likely maintains ECM flexibility and allows adipocytes to grow larger, without the mechanical tension and rupture that contribute to inflammation in obese adipose tissue.

Blockade of cadherin-11 significantly improves glycemic control in obese WT mice. The remarkable protection from HFD-induced glucose intolerance in cad-11/- mice led us to consider whether targeting stromal cell cadherin-11 might offer a new therapeutic approach for obesity-induced glucose intolerance. Thus, we investigated the therapeutic potential of anti-cadherin-11blocking mAbs to improve glucose tolerance in obesity. HFD-fed WT mice were divided into 2 equal groups on the basis of their BWs (Figure 7A and Supplemental Figure 7A). Compared with isotype control mouse IgG1 (mIgG1) Ab treatment, 2 or 3 weeks of anticadherin-11-specific Ab (SYN12) treatment in HFD-fed WT mice significantly improved glucose tolerance, as determined by GTT and AUC analysis (Figure 7B and Supplemental Figure 7B). Anticadherin-11 Ab treatment did not alter body or fat pad weights in WT mice on a HFD (Figure 7A and Supplemental Figure 7A), which confirmed that the improved glucose tolerance in both cad-11/- mice and anti-cadherin-11-treated WT mice was independent of BW. Consistent with the results in obese cad-11/-- mice, anti-cadherin- $11 \mathrm{Ab}$ treatment in obese mice also revealed a trend toward increased levels of Il33 and significantly increased levels of Il13, which are known to drive ILC2 and M2 macrophages, along with decreased Col6a3 expression in adipose tissue compared with levels detected in control obese WT mice (Figure 7, C-E). Further, anti-cadherin-11 Ab treatment significantly decreased liver weights in obese WT mice (Figure 7F). Importantly, these 2- to 3-week-long Ab treatment studies suggested that the beneficial metabolic phenotype is mediated by the interference of cadherin-11 interactions rather than by developmental effects in cad- $11^{-/-}$mice. Thus, cadherin-11 may be useful as a stromal cell therapeutic target for treating adipose tissue inflammation, glucose intolerance, and metabolic syndrome in obesity.

\section{Discussion}

Fibroblasts have been implicated in the regulation of immune responses (26). For example, fibroblastic reticular cells in lymph nodes suppress $\mathrm{T}$ cell activation and expansion to maintain peripheral tolerance (21-23). Fibroblasts also modulate inflammation such as that seen with inflammatory arthritis, psoriasis, inflammatory bowel disease, and cancer by recruiting, activating, inhibiting, or maintaining leukocytes in tissues $(24,36,37)$. Importantly, 
inflammation plays a causative role in adipose tissue dysfunction and obesity-induced insulin resistance $(1-4,38,39)$. Fibroblast populations including adipocyte precursors are relatively high and account for over $90 \%$ of nonhematopoietic stromal vascular cells in adipose tissue. However, the of role stromal fibroblasts in adipose tissue inflammation and diabetes is unknown.

Here, we show that fibroblast function can be modified by either the deficiency or the blockade of mesenchymal cell cadherin11. By targeting cadherin-11, we found that fibroblasts play a key role in adipose tissue inflammation and glucose intolerance in obesity. Cadherin-11 deficiency strikingly prevented obese mice from adipose tissue inflammation and metabolic syndrome. Adipose tissue inflammation is accompanied by a phenotypic switch from M2 antiinflammatory to M1 proinflammatory macrophages $(3,4,40)$. However, despite their obesity, we found that adipose tissue from cad-11/-- mice contained significantly higher numbers of M2 macrophages than did WT mice. We also found that IL-33-mediated ILC2 activation resulted in an increase in IL-13 expression that maintained M2 macrophage phenotypes in adipose tissue of obese cad-11/-- mice.

In obesity, IL-33 plays a protective role in adipose tissue inflammation and insulin resistance $(9,41)$. IL-33 induces the homeostasis and function of ST2-expressing ILC2s and Tregs (9, 42). However, the source of IL-33 in adipose tissue was unclear. Studies suggested that IL-33 is mainly produced by stromal cells such as epithelial or endothelial cells (43-45). In our study, we found that the levels of IL-33 were much higher in stromal vascular cells than in adipocytes and that IL-33 expression by stromal vascular cells, but not adipocytes, was increased with cadherin-11 deficiency. Furthermore, we found that among SVF cells, PDGFR $\alpha^{+}$fibroblasts were the major cells producing IL-33. Some PDGFR $\alpha^{-}$stromal vascular cells also displayed IL-33 expression, and these stromal vascular cells may include endothelial cells (43) and fibroblasts expressing low levels of surface PDGFR $\alpha$. Compared with PDGFR $\alpha^{+}$fibroblasts, which constitute over $90 \%$ of nonhematopoietic cells in SVF cells, the percentage of PDGFR $\alpha$ stromal vascular cells is much smaller, and thus these cells may not contribute significantly to the production of IL-33 in adipose tissue. IL-33 expression was also detected by immunofluorescence in cadherin-11-expressing cells in adipose tissue of lean mice in a recent study (14), further supporting our finding that PDGFR $\alpha^{+}$ fibroblasts are the major cells expressing IL-33 in adipose tissue.

A recent report showed that expansion of adipocyte precursor cells occurs within the first week of HFD feeding and that their numbers return to basal levels over the course of a 6-week HFD (46). The rapid proliferation of adipocyte precursors is mediated through activation of the PI3K/AKT2 pathway (46). Interestingly, compared with WT fibroblasts, cad-11 ${ }^{-/-}$fibroblasts showed a markedly higher proliferation rate during the first week of a HFD, with their numbers returning to basal levels by week 5 of the HFD. Data from the Immunological Genome Project (ImmGen) showed that, among PDGF ligands ( $P d g f a, P d g f b, P d g f c$, and $P d g f d), P d g f c$ expression is particularly high in adipose tissue macrophages compared with expression levels in macrophages in other tissues. The accu-

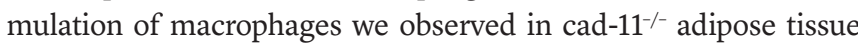
may have provided higher levels of $P d g f c$, thereby driving the subsequent proliferation of PDGFR $\alpha^{+}$fibroblasts in the adipose tissue of HFD-fed cad- $11^{-1-}$ mice. In addition, we found that the healthy adipocytes in obese cad-11-/- mice allowed adipose tissue to store larger amounts of lipids, which may have reduced obesogenic adipogenesis. Further, we show that the higher IL-33 expression in cad- $11^{-/}$adipose tissue resulted from an increase in the number of PDGFR $\alpha^{+}$fibroblasts. In turn, IL-33 expression by the expanded PDGFR $\alpha^{+}$fibroblasts in cad-11/-- adipose tissue in the first week of the HFD may enhance M2 macrophage differentiation through ILC2-produced IL-13. Therefore, the early response of cad-11/mice to a HFD appears to determine the Th2-type antiinflammatory adipose tissue microenvironment, which maintains adipose tissue function and reduces obesity-induced adipose tissue inflammation. Such rapid antiinflammatory mechanisms may also prevent glucose intolerance following binge eating by animals such as predators in the wild as well as in humans experiencing overnutrition.

Obesity-induced adipocyte hypertrophy and hyperplasia require extensive adipose tissue remodeling. ECM proteins and their turnover are essential for both the development and remodeling of adipose tissue $(34,35)$. Less rigid ECM in WAT better accommodates adipocyte enlargement, with less adipocyte death and inflammation in obesity $(16,34)$. Fibrosis contributes to adipose tissue dysfunction and has a causative role in insulin resistance in obesity $(1,16,39,47,48)$. A recent study showed that COL6deficient mice had less adipocyte death and adipose tissue fibrosis, which were associated with improved glucose metabolism in both HFD-induced and genetically obese mice (34). Importantly, we found that cadherin-11 deficiency reduced the production of collagens COL3 and COL6 by fibroblasts and resulted in substantially less adipose tissue fibrosis in obesity. Anti-cadherin-11 mAb blockade also markedly reduced COL6a3 production. These data suggest that cadherin-11 regulates collagen production by adipose tissue fibroblasts, and this may help to preserve adipose tissue flexibility, larger adipocytes, and reduced adipocyte necrosis and inflammation crown-like structures in obese cad-11/mice. In addition, cadherin-11 blockade may be relevant to other diseases linked by ECM deposition by cadherin-11-expressing fibroblasts or myofibroblasts. A recent study showed that obesity increases myofibroblasts and ECM stiffness in the adipose tissue of mammary fat and that changes in ECM mechanics promote breast tumorigenesis in obesity (49). It would be interesting to determine whether blocking cadherin-11 in the myofibroblasts of mammary fat can reduce the risk for breast tumorigenesis by inhibiting obesity-induced collagen production.

In summary, cad-11 ${ }^{-/}$mice are protected from obesityinduced glucose intolerance and fatty liver as a result of reduced adipose tissue inflammation associated with the axis of stromal cell IL-33, ILC2s, and M2 macrophages, as well as less adipose tissue fibrosis. Fibroblast stromal cells expressing cadherin-11 play a major role in regulating the activation of innate immune cells and adipose tissue matrix stiffness. These findings strongly point to the importance of mesenchymal stromal cells and the opportunity to target them via cadherin- 11 in the management of adipose tissue inflammation, diabetes, and metabolic syndrome.

\section{Methods}

Animal studies. The cad-11//- mice and control B6:129 F1-intercrossed mice were maintained at Taconic. Littermates of WT and cad-11/mice were bred and maintained at Taconic. WT C57BL/6 mice were 
purchased from Taconic. When needed, mice were shipped to the Dana-Farber mouse facility and maintained in the same room during the experiments. Male mice, 6 to 8 weeks of age, were given a HFD, which consisted of $60 \% \mathrm{kcal}$ from fat (D12492; Research Diets), or a ND (ProLab IsoPro RMH 3000; LabDiet) for 5 to 12 weeks as indicated in each figure legend. For GTTs, mice were fasted for 6 hours or overnight for 10 hours and given i.p. D-glucose $(1 \mathrm{~g}$ glucose per $1 \mathrm{~kg}$ BW). Blood glucose levels were determined at $0,15,30,60$, and 90 minutes after glucose injection using the OneTouch Ultra Glucometer (LifeScan Inc.). ITTs were performed after a 4-hour morning fast and i.p. injection of insulin (0.75 IU/kg BW) (HumilinR $100 \mathrm{U} / \mathrm{ml}$; Eli Lilly). Blood glucose levels were measured at $0,15,30,60$, and $90 \mathrm{~min}$ utes. The HOMA-IR index was calculated as follows: insulin $(\mu \mathrm{IU} / \mathrm{ml}) \times$ glucose $(\mathrm{mg} / \mathrm{dl}) / 405$. For Ab treatment experiments, B6 mice were fed a HFD for 6 weeks. mIgG1 isotype control Ab or anti-cadherin-11specific $\mathrm{mAb}$ (SYN12) was given i.p. to HFD-fed mice at $10 \mathrm{mg} / \mathrm{kg} \mathrm{BW}$ every 3 days for 3 weeks. For the Ab experiments in Supplemental Figure 3, Ab treatment was initiated on B6 mice fed a HFD for 3 weeks. mIgG1 or anti-cadherin-11 mAb (SYN12) was given i.p. at $500 \mu \mathrm{g}$ per mouse for the first dose and then at $100 \mu \mathrm{g}$ per mouse every 3 days for 2 weeks. Body composition was analyzed in conscious mice using an EchoMRI 3-in-1 analyzer.

Adipose tissue digestion. Adipose tissue was cut into small pieces and digested by enzyme mixture (RPMI 1640 medium containing 1 $\mathrm{mg} / \mathrm{ml}$ collagenase type 2 [LSO04188; Worthington Biochemical]; $0.1 \mathrm{mg} / \mathrm{ml}$ DNase I [10104159001; Roche]; and $0.8 \mathrm{mg} / \mathrm{ml}$ Dispase II [04942078001; Roche]) for 1 hour at $37^{\circ} \mathrm{C}$. During digestion, released cells were collected on ice, and fresh enzyme mixture was added every 10 minutes. Using this method, adipose tissue was completely digested to release stromal cells including fibroblasts. Released cells were spun down to separate SVF cells from floating adipocytes. Stromal vascular cells were resuspended in complete RPMI 1640 medium containing 10\% FBS and filtered through a $70-\mu \mathrm{m}$ filter. Total live cell numbers in the SVF were counted using a trypan blue exclusion test.

Flow cytometric analysis. Cells were incubated with Zombie Aqua (1:100; BioLegend) in PBS at room temperature for 5 minutes. After washing, cells were resuspended in FACS buffer (2\% FBS, 2 mM EDTA, $0.05 \% \mathrm{NaN}_{3}$ in PBS) and incubated with anti-CD16/32 Abs in FACS buffer for 10 minutes on ice, followed by staining with the Abs indicated in figures in FACS buffer for 15 minutes at $4^{\circ} \mathrm{C}$. After washing, the stained cells were analyzed using a FACSCanto II System (BD Biosciences). Data were analyzed with FlowJo and FCS Express 5 (De Novo Software) software. For cadherin-11 detection, stromal vascular cells were stained with Zombie Aqua as described above, except $2 \mathrm{mM} \mathrm{CaCl}_{2}$ in HEPESbuffered saline (HBS-Ca ${ }^{++}$) was used instead of PBS. After washing, Fc receptors were blocked with anti-CD16/32 Abs and then incubated with biotinylated anti-cadherin-11 $\mathrm{Ab}(0.5 \mu \mathrm{g})$ for 20 minutes on ice in HBS buffer $\left(2 \% \mathrm{FBS}\right.$ in $\left.\mathrm{HBS}^{-\mathrm{Ca}^{++}}\right)$to minimize cadherin proteolysis. After washing, the cells were stained with APC-conjugated streptavidin and other fluorescence-labeled Abs against surface markers (Ter119, CD45, CD31, Sca-1, and PDGFR $\alpha$ ). Cadherin-11 expression was analyzed on hematopoietic cells (CD45 ${ }^{+}$Ter119-) and fibroblast stromal cells (CD45-Ter119CD31-Sca-1 $1^{+} \mathrm{PDGFR} \alpha^{+}$) after excluding dead cells and doublets.

Immunofluorescence. For whole fat tissue staining, $5 \mathrm{~mm}^{2}$ of tissue was fixed in $4 \%$ paraformaldehyde in PBS overnight, washed in PBS for 2 days at $4^{\circ} \mathrm{C}$, and permeabilized and blocked in blocking buffer
(5\% serum, $1 \% \mathrm{BSA}$, and $1 \%$ Triton $\mathrm{X}-100$ in PBS) overnight at $4^{\circ} \mathrm{C}$. Then, tissues were stained with the indicated $\mathrm{Ab}$ below in blocking buffer for 2 days and washed in blocking buffer for 1 day at $4^{\circ} \mathrm{C}$, followed by staining with Alexa Fluor 488-conjugated goat anti-mouse CD68, Alexa Fluor 568 phalloidin (F-actin), and DRAQ5 (nucleus) in blocking buffer overnight at $4^{\circ} \mathrm{C}$. After washing tissue pieces for 3 days at $4^{\circ} \mathrm{C}$, images were taken by confocal microscopy (TE2000-U; Nikon). For cadherin-11 detection in ex vivo stromal vascular cell cultures, freshly released SVF cells were seeded on a glass coverslip in DMEM medium containing 10\% FBS, 2 mM L-glutamine, 100 units/ $\mathrm{ml}$ penicillin, $100 \mu \mathrm{g} / \mathrm{ml}$ streptomycin sulfate, $10 \mu \mathrm{g} / \mathrm{ml}$ gentamycin, and $55 \mu \mathrm{M}$ 2-mercaptoethanol (Gibco-BRL, Thermo Fisher Scientific) at $37^{\circ} \mathrm{C}$ under $10 \% \mathrm{CO}_{2}$. After 2 days of culture, cells were fixed in $4 \%$ paraformaldehyde in $\mathrm{HBS}-\mathrm{Ca}^{++}$for 20 minutes at room temperature and washed 4 times for 5 minutes each. Cells were then permeabilized in $0.2 \%$ Triton $\mathrm{X}-100$ in $\mathrm{HBS}-\mathrm{Ca}^{++}$for 3 minutes and then washed twice for 5 minutes each. After treatment with blocking buffer (HBS$\mathrm{Ca}^{++}$containing $1 \% \mathrm{BSA}$ and $10 \% \mathrm{FBS}$ ) for 1 hour at room temperature, cells were incubated with anti-cadherin- $11 \mathrm{Ab}(1: 500 ; 3 \mathrm{H} 10$, in-house) in blocking buffer overnight at $4^{\circ} \mathrm{C}$. After dip-washing 6 times, cells were stained with Alexa Fluor 488-conjugated goat anti-mouse Ab (1:750); Alexa Fluor 568 phalloidin (1:500); and DRAQ5 (1:500) for 1 hour at room temperature. Cells were washed twice with blocking buffer and twice with $\mathrm{HBS}-\mathrm{Ca}^{++}$for 5 minutes each. Images were taken by confocal microscopy.

ELISA. Mouse serum was analyzed for insulin (90080; CRYSTAL CHEM Inc.); adiponectin (EZMADP-60K; EMD Millipore); FGF21 (MF2100; R\&D Systems); TG (290-63701; Wako); and FFA (ab65341; Abcam) following the manufacturers' protocols. For IL-33 ELISA, harvested fat tissue was immediately frozen in $\mathrm{N}_{2}$ gas and stored at $-80^{\circ} \mathrm{C}$ until use. The frozen tissue was placed in PBS containing 1\% Triton X-100 and protease inhibitors (11873580001; Roche) and homogenized with a tissue dissociator. After incubating homogenates for 20 minutes at $4^{\circ} \mathrm{C}$, tissue lysates were cleared by spinning at $10,000 \mathrm{~g}$ for 15 minutes. Soluble lysates were analyzed for IL-33 by ELISA (M3300; R\&D Systems). IL-33 levels were normalized to the total protein concentration in the tissue lysates.

Immunoblotting. After HFD feeding for 12 weeks, WT and cad-11 ${ }^{-1-}$ mice were fasted for 6 hours, and $20 \mathrm{U} / \mathrm{kg}$ insulin was injected via the inferior vena cava. Three minutes later, tissues were taken and homogenized in lysis buffer (1\% Triton X-100, $50 \mathrm{mM}$ Tris $\mathrm{HCl}, 150 \mathrm{mM}$ $\mathrm{NaCl}, 5 \mathrm{mM}$ EDTA, $1 \mathrm{mM}$ PMSF) with protease cocktail (Roche) and phosphatase inhibitors $\left(1 \mathrm{mM} \mathrm{Na}_{3} \mathrm{VO}_{4}, 10 \mathrm{mM} \mathrm{NaF}\right)$. Total lysates were cleared, and lysates $(50 \mu \mathrm{g})$ were loaded into each lane. The membrane was blotted with 1:2,500 anti-total AKT or anti-p-AKT Ab, washed with TBS-Tween-20 (TBST), and incubated with 1:7,500 donkey HRPconjugated anti-rabbit $\mathrm{Ab}$. The protein in the membrane was visualized using Bio-Rad Clarity Western ECL Substrate (1705060; Bio-Rad).

$A b s$ and reagents. For macrophage staining, the following Abs were used: eFluor 450-CD45 (30-F11, 48-0451; eBioscience); APCeFluor 780-Ter119 (Ter119, 47-5921-82; eBioscience); PerCP/ Cy5.5-F480 (BM8, 557397; BD); PE-CD11b (M1/70, 45-4801-80; eBioscience); APC-CD11c (HL3, 550261; BD); and FITC-CD206 (C068C2, 141704; BioLegend). For cadherin-11 staining in mice, the following Abs were used: PE-Cy7-CD45 (30-F11, 25-0451-82; eBioscience); APC- eFluor 780-Ter119 (Ter119, 47-5921-82; eBioscience); Pacific Blue-CD31 (390, 48-0311-82; eBioscience); PE-PDGFR1a (APA5, 
12-1401-81; eBioscience); Alexa Fluor 488-Ly-6A/E (Sca-1) (D7, 108116; BioLegend); biotinylated anti-cadherin-11 (in-house); and streptavidinAPC (016-130-084; Jackson ImmunoResearch). For cadherin11 staining in humans, the following Abs were used: BV421-CD45 (Hl30, 563879; BD); APC-Alexa Fluor 750-CD235a (A89314; Beckman Coulter); PE-Cy7-CD31 (WM-59, 25-0319; eBioscience); FITC-CD90 (555595; BD); and biotinylated anti-cadherin-11 (in-house). Dead cells were identified by staining with Zombie Aqua (423101; BioLegend). For immunofluorescence, the following Abs were used: CD68 (FA-11; BioLegend); cadherin-11 (16A, MAB2014; EMD Millipore or 3H10, in-house); Alexa Fluor 488-conjugated goat anti-mouse (115-546-062; The Jackson Laboratory); Alexa Fluor 568 phalloidin (A12380; Invitrogen, Thermo Fisher Scientific); and DRAQ 5 (4084; Cell Signaling Technology). For immunoblotting, total AKT (C67E7, 4691; Cell Signaling Technology) and p-AKT (Ser473) (D9E, 4060; Cell Signaling Technology) were used.

$q P C R$ analysis. Tissues were snap-frozen in nitrogen gas and stored at $-80^{\circ} \mathrm{C}$ until use. Fat, muscle, and liver tissues were homogenized in TRIzol Reagent (15596026; Life Technologies, Thermo Fisher Scientific) and mixed with chloroform (TRIzol/chloroform ratio of 5:1). After spinning down, the upper aqueous phase was mixed with the same volume of $70 \% \mathrm{EtOH}$ and applied to the RNeasy Mini Kit column according to the procedure for the RNeasy Mini Kit (74104; QIAGEN). Isolated RNA was converted to cDNA (QuantiTect Reverse Transcription Kit, 205311; QIAGEN) according to the manufacturer's instructions. qPCR (Brilliant III Ultra-Fast SYBR Green QPCR Master Mix, 600882; Agilent Technologies) was performed with gene-specific primers. The following mouse primers (forward and reverse sequences)were used: Gapdh: CATCACTGCCACCCAGAAGACTG and ATGCCAGTGAGCTTCCCGTTCAG; Cdh11 GCCAACAGCCCAATAAGGTA and CTGGGACTTTGGTTTCCTGA; Il33: CTACTGCATGAGACTCCGTTCTG and AGAATCCCGTGGATAGGCAGAG; Tslp: GCAAATCGAGGACTGTGAGAGC and TGAGGGCTTCTCTTGTTCTCCG; Il25: TGGCTGAAGTGGAGCTCTGCAT and CCCGATTCAAGTCCCTGTCCAA; Il5: GATGAGGCTTCCTGTCCCTACT and TGACAGGTTTTGGAATAGCATTTCC; Il13: AACGGCAGCATGGTATGgAgtg and TGgGtCCTGtAgAtgGCATtGC; Fgf21: CTCTAGGTTTCTTTGCCAACAG and AAGCTGCAGGCCTCAGGAT; Adiopq: AGATGGCACTCCTGGAGAGAAG and ACATAAGCGGCTTCTCCAGGCT; Col1a1: CCTCAGGGTATTGCTGGACAAC and CAGAAGGACCTTGTTTGCCAGG; Col2a1: GCTGGTGAAGAAGGCAAACGAG and CCATCTTGACCTGGGAATCCAC; Col3a1: GACCAAAAGGTGATGCTGGACAG and CAAGACCTCGTGCTCCAGTTAG; Col4a1: ATGGCTTGCCTGGAGAGATAGG and TGgtTGCCCTtTGAgtCCTGGA; Col5a1: AGATGGCATCCGAGGTCTGAAG and GACCTTCAGGACCATCTTCTCC; Col6a1: GACACCTCTCAGTGTGCTCTGT and GCGATAAGCCTTGGCAGGAAATG; Col6a2: TGGTCAACAGGCTAGGTGCCAT and TAGACAGGGAGTTGACTCGCTC; Col6a3: CAGAACCATTGTTTCTCACT and AGGACTACACATCTTTTCAC; Arg1: CATTGGCTTGCGAGACGTAGAC and GCTGAAGGTCTCTTCCATCACC; Mrc1: GTTCACCTGGAGTGATGGTTCTC and AGGACATGCCAGGGTCACCTTT; Il10: AATAAGCTCCAAGACCAAGG and CAGACTCAATACACACTG; Tnf: CTCTTCTGCCTGCTGCACTTTG and ATGGGCTACAGGCTTGTCACTC; iNOs: GAGACAGGGAAGTCTGAAGCAC and CCAGCAGTAGTTGCTCCTCTTC. $M C P-1$ primer sets were purchased from SA Biosciences.

Nonpolar lipid extractions and TLC analysis. Hepatic nonpolar lipids were extracted using a modified Folch method (50). Briefly, individual livers were suspended in 2 volumes of 10:1 $\mathrm{CH}_{3} \mathrm{OH} / \mathrm{O} .3 \% \mathrm{NaCl}$ $(\mathrm{v} / \mathrm{v})$ and biphased against 2 volumes of petroleum ether. Organs were homogenized with a glass rod and the mixture vortexed. The upper phase containing nonpolar lipids was collected, and biphasing of liver homogenates was repeated an additional 2 times. The petroleum ether fractions were dried to recover nonpolar lipids. For normal-phase TLC, the mobile phase was 90:10:3 hexane/diethyl ether/formic acid (v/v/v). Lipids were visualized with $5 \%$ molybdophosphoric acid (SigmaAldrich) followed by charring. HPLC-grade hexane, diethyl ether, and formic acid were from purchased from Thermo Fisher Scientific. Aluminum TLC silica gel 60 plates $(10-\mathrm{cm})$ were used (EMD Millipore).

Statistics. All data are presented as the mean \pm SEM. Statistical significance was determined using a Student's 2-tailed $t$ test or 2-way ANOVA. A $P$ value of less than 0.05 was considered statistically significant. GraphPad Prism 5 (GraphPad Software) was used for all statistical analyses.

Study approval. Animal studies were approved by the Dana-Farber Cancer Institute animal care and use committee. Discarded visceral adipose tissues from obese patients were obtained with IRB approval of Brigham and Women's Hospital.

\section{Author contributions}

SKC and MBB conceived and designed the experiments and wrote the manuscript. SKC, ACK, BJW, FM, KW, PYL, DD, LL, and VCM performed the experiments. XM performed and analyzed the confocal images. ASB critically reviewed the manuscript.

\section{Acknowledgments}

We thank Steven E. Shoelson at the Joslin Diabetes Center (Boston, Massachusetts, USA) and Hyun Cheol Roh at Beth Israel Deaconess Medical Center (Boston, Massachusetts, USA) for their helpful discussions. We also thank Adam T. Chicoine, manager of the flow cytometry core at Brigham and Women's Hospital Human Immunology Center for Flow Sorting. This research was supported by NIH grants R01 AR063709 and R01 AI113046 (to MBB).

Address correspondence to: Michael B. Brenner, Theodore B. Bayles Professor of Medicine, Harvard Medical School, Chief, Division of Rheumatology, Immunology and Allergy, Brigham and Women's Hospital, Building For Transformative Medicine, Room 6002, 60 Fenwood Road, Boston, Massachusetts 02115, USA. Phone: 617.525.1000; Email: mbrenner@research.bwh.harvard.edu.

SKC's present address is: JW CreaGene Corporation, Seongnamsi, Gyeonggi-do, 13202, South Korea.
1. Johnson AM, Olefsky JM. The origins and drivers of insulin resistance. Cell. 2013;152(4):673-684.

2. Hotamisligil GS, Shargill NS, Spiegelman BM. Adipose expression of tumor necrosis factor- alpha: direct role in obesity-linked insulin resistance. Science. 1993;259(5091):87-91.

3. Weisberg SP, McCann D, Desai M, Rosenbaum M, Leibel RL, Ferrante AW. Obesity is associated with macrophage accumulation in adipose tissue. JClin Invest. 2003;112(12):1796-1808.

4. $\mathrm{Xu} \mathrm{H}$, et al. Chronic inflammation in fat plays a crucial role in the development of obesi- 
ty-related insulin resistance. JClin Invest. 2003;112(12):1821-1830.

5. Wynn TA, Chawla A, Pollard JW. Macrophage biology in development, homeostasis and disease. Nature. 2013;496(7446):445-455.

6. Han MS, et al. JNK expression by macrophages promotes obesity-induced insulin resistance and inflammation. Science. 2013;339(6116):218-222.

7. Chawla A, Nguyen KD, Goh YP. Macrophagemediated inflammation in metabolic disease. Nat Rev Immunol. 2011;11(11):738-749.

8. Wu D, et al. Eosinophils sustain adipose alternatively activated macrophages associated with glucose homeostasis. Science. 2011;332(6026):243-247.

9. Molofsky AB, et al. Innate lymphoid type 2 cells sustain visceral adipose tissue eosinophils and alternatively activated macrophages. J Exp Med. 2013;210(3):535-549.

10. Brestoff JR, et al. Group 2 innate lymphoid cells promote beiging of white adipose tissue and limit obesity. Nature. 2015;519(7542):242-246.

11. Lee MW, et al. Activated type 2 innate lymphoid cells regulate beige fat biogenesis. Cell. 2015;160(1-2):74-87.

12. Price AE, et al. Systemically dispersed innate IL-13-expressing cells in type 2 immunity. Proc Natl Acad Sci USA. 2010;107(25):11489-11494.

13. Vasanthakumar A, et al. The transcriptional regulators IRF 4, BATF and IL-33 orchestrate development and maintenance of adipose tissue-resident regulatory T cells. Nat Immunol. 2015;16(3):276-285.

14. Kolodin D, et al. Antigen- and cytokine-driven accumulation of regulatory $\mathrm{T}$ cells in visceral adipose tissue of lean mice. Cell Metab. 2015;21(4):543-557.

15. Miller AM, et al. Interleukin-33 induces protective effects in adipose tissue inflammation during obesity in mice. Circ Res. 2010;107(5):650-658.

16. Sun K, Tordjman J, Clément K, Scherer PE. Fibrosis and adipose tissue dysfunction. Cell Metab. 2013;18(4):470-477.

17. Wynn TA. Integrating mechanisms of pulmonary fibrosis. JExp Med. 2011;208(7):1339-1350.

18. Lee DM, et al. Cadherin-11 in synovial lining formation and pathology in arthritis. Science. 2007;315(5814):1006-1010.

19. Luther SA, Tang HL, Hyman PL, Farr AG, Cyster JG. Coexpression of the chemokines ELC and SLC by T zone stromal cells and deletion of the ELC gene in the plt/plt mouse. Proc Natl Acad Sci USA. 2000;97(23):12694-12699.

20. Link A, et al. Fibroblastic reticular cells in lymph nodes regulate the homeostasis of naive $\mathrm{T}$ cells.
Nat Immunol. 2007;8(11):1255-1265.

21. Siegert S, et al. Fibroblastic reticular cells from lymph nodes attenuate $\mathrm{T}$ cell expansion by producing nitric oxide. PLoS One. 2011;6(11):e27618

22. Lukacs-Kornek V, et al. Regulated release of nitric oxide by nonhematopoietic stroma controls expansion of the activated $\mathrm{T}$ cell pool in lymph nodes. Nat Immunol. 2011;12(11):1096-1104.

23. Khan O, Headley M, Gerard A, Wei W, Liu L, Krummel MF. Regulation of $\mathrm{T}$ cell priming by lymphoid stroma. PLoS One. 2011;6(11):e26138.

24. Chang SK, et al. Cadherin-11 regulates fibroblast inflammation. Proc Natl Acad Sci USA. 2011;108(20):8402-8407.

25. Guerne PA, Zuraw BL, Vaughan JH, Carson DA, Lotz M. Synovium as a source of interleukin 6 in vitro. Contribution to local and systemic manifestations of arthritis. JClin Invest. 1989;83(2):585-592.

26. Buckley $C D$. Why does chronic inflammation persist: An unexpected role for fibroblasts. Immunol Lett. 2011;138(1):12-14.

27. Takeichi M. Cadherins: a molecular family important in selective cell-cell adhesion. Annu Rev Biochem. 1990;59:237-252.

28. Patel SD, et al. Type II cadherin ectodomain structures: implications for classical cadherin specificity. Cell. 2006;124(6):1255-1268.

29. Wu M, et al. Identification of cadherin 11 as a mediator of dermal fibrosis and possible role in systemic sclerosis. Arthritis Rheumatol. 2014;66(4):1010-1021.

30. Schneider DJ, et al. Cadherin-11 contributes to pulmonary fibrosis: potential role in TGF- $\beta$ production and epithelial to mesenchymal transition. FASEB J. 2012;26(2):503-512.

31. Vandooren B, et al. Tumor necrosis factor alpha drives cadherin 11 expression in rheumatoid inflammation. Arthritis Rheum. 2008;58(10):3051-3062.

32. Valencia X, et al. Cadherin-11 provides specific cellular adhesion between fibroblast-like synoviocytes. JExp Med. 2004;200(12):1673-1679.

33. Zeyda $\mathrm{M}$, et al. Severe obesity increases adipose tissue expression of interleukin-33 and its receptor ST2, both predominantly detectable in endothelial cells of human adipose tissue. Int JObes (Lond). 2013;37(5):658-665.

34. Khan T, et al. Metabolic dysregulation and adipose tissue fibrosis: role of collagen VI. Mol Cell Biol. 2009;29(6):1575-1591.

35. Chun TH, Hotary KB, Sabeh F, Saltiel AR, Allen ED, Weiss SJ. A pericellular collagenase directs the 3-dimensional development of white adipose tissue. Cell. 2006;125(3):577-591.

36. Kalluri R, Zeisberg M. Fibroblasts in cancer. Nat
Rev Cancer. 2006;6(5):392-401.

37. Filer A, Pitzalis C, Buckley CD. Targeting the stromal microenvironment in chronic inflammation. Curr Opin Pharmacol. 2006;6(4):393-400.

38. Gregor MF, Hotamisligil GS. Inflammatory mechanisms in obesity. Annu Rev Immunol. 2011;29:415-445.

39. Odegaard JI, Chawla A. Pleiotropic actions of insulin resistance and inflammation in metabolic homeostasis. Science. 2013;339(6116):172-177.

40. Lumeng CN, Bodzin JL, Saltiel AR. Obesity induces a phenotypic switch in adipose tissue macrophage polarization. JClin Invest. 2007;117(1):175-184.

41. Miller AM, et al. Interleukin-33 induces protective effects in adipose tissue inflammation during obesity in mice. Circ Res. 2010;107(5):650-658.

42. Han JM, Wu D, Denroche HC, Yao Y, Verchere CB, Levings MK. IL-33 Reverses an obesityinduced deficit in visceral adipose tissue ST2+ T regulatory cells and ameliorates adipose tissue inflammation and insulin resistance. JImmunol. 2015;194(10):4777-4783.

43. Zeyda M, et al. Severe obesity increases adipose tissue expression of interleukin-33 and its receptor ST2, both predominantly detectable in endothelial cells of human adipose tissue. Int JObes (Lond). 2013;37(5):658-665.

44. Pichery M, et al. Endogenous IL-33 is highly expressed in mouse epithelial barrier tissues, lymphoid organs, brain, embryos, and inflamed tissues: in situ analysis using a novel Il-33LacZ gene trap reporter strain. JImmunol. 2012;188(7):3488-3495.

45. Liew FY, Pitman NI, McInnes IB. Diseaseassociated functions of IL-33: the new kid in the IL-1 family. Nat Rev Immunol.2010;10(2):103-110.

46. Jeffery E, Church CD, Holtrup B, Colman L, Rodeheffer MS. Rapid depot-specific activation of adipocyte precursor cells at the onset of obesity. Nat Cell Biol. 2015;17(4):376-385.

47. Lumeng CN, Saltiel AR. Inflammatory links between obesity and metabolic disease. JClin Invest. 2011;121(6):2111-2117.

48. Osborn O, Olefsky JM. The cellular and signaling networks linking the immune system and metabolism in disease. Nat Med. 2012;18(3):363-374.

49. Seo BR, et al. Obesity-dependent changes in interstitial ECM mechanics promote breast tumorigenesis. Sci Transl Med. 2015;7(301):301ra130.

50. Tatituri RV, et al. Inactivation of Corynebacterium glutamicum NCgl0452 and the role of MgtA in the biosynthesis of a novel mannosylated glycolipid involved in lipomannan biosynthesis. JBiol Chem. 2007;282(7):4561-4572. 